\title{
Longitudinal data in peripheral blood confirm that PM20D1 is a quantitative trait locus (QTL) for Alzheimer's disease and implicate its dynamic role in disease progression
}

Qi Wang ${ }^{1 *} \mathbb{D}$, Yinghua Chen ${ }^{2}$, Benjamin Readhead ${ }^{1}$, Kewei Chen $^{2}$, Yi Su$^{2}$, Eric M. Reiman ${ }^{1,2}$ and Joel T. Dudley ${ }^{1,3}$

\begin{abstract}
Background: While Alzheimer's disease (AD) remains one of the most challenging diseases to tackle, genome-wide genetic/epigenetic studies reveal many disease-associated risk loci, which sheds new light onto disease heritability, provides novel insights to understand its underlying mechanism and potentially offers easily measurable biomarkers for early diagnosis and intervention.

Methods: We analyzed whole-genome DNA methylation data collected from peripheral blood in a cohort $(n=649)$ from the Alzheimer's Disease Neuroimaging Initiative (ADNI) and compared the DNA methylation level at baseline among participants diagnosed with $\mathrm{AD}(n=87)$, mild cognitive impairment $(\mathrm{MCl}, n=175)$ and normal controls ( $n=162$ ), to identify differentially methylated regions (DMRs). We also leveraged up to 4 years of longitudinal DNA methylation data, sampled at approximately 1 year intervals to model alterations in methylation levels at DMRs to delineate methylation changes associated with aging and disease progression, by linear mixed-effects (LME) modeling for the unchanged diagnosis groups (AD, MCl and control, respectively) and U-shape testing for those with changed diagnosis (converters).

Results: When compared with controls, patients with $\mathrm{MCl}$ consistently displayed promoter hypomethylation at methylation QTL ( $\mathrm{mQTL}$ ) gene locus PM20D1. This promoter hypomethylation was even more prominent in patients with mild to moderate AD. This is in stark contrast with previously reported hypermethylation in hippocampal and frontal cortex brain tissues in patients with advanced-stage AD at this locus. From longitudinal data, we show that initial promoter hypomethylation of PM20D1 during MCl and early stage AD is reversed to eventual promoter hypermethylation in late stage $A D$, which helps to complete a fuller picture of methylation dynamics. We also confirm this observation in an independent cohort from the Religious Orders Study and Memory and Aging Project (ROSMAP)
\end{abstract}

\footnotetext{
*Correspondence: Qi.Wang.10@asu.edu

${ }^{1}$ ASU-Banner Neurodegenerative Disease Research Center, Arizona State University, Tempe, AZ, USA

Full list of author information is available at the end of the article

Qi Wang: Data used in preparation of this article were obtained from the Alzheimer's Disease Neuroimaging Initiative (ADNI) database (adni.loni. usc.edu). As such, the investigators within the ADNI contributed to the design and implementation of ADNI and/or provided data but did not participate in analysis or writing of this report. A complete listing of ADNI investigators can be found at: http://adni.loni.usc.edu/wpcontent/uploa ds/how_to_apply/ADNI_Acknowledgement_List.pdf.
} permits use, sharing, adaptation, distribution and reproduction in any medium or format, as long as you give appropriate credit to the original author(s) and the source, provide a link to the Creative Commons licence, and indicate if changes were made. The images or other third party material in this article are included in the article's Creative Commons licence, unless indicated otherwise in a credit line to the material. If material is not included in the article's Creative Commons licence and your intended use is not permitted by statutory regulation or exceeds the permitted use, you will need to obtain permission directly from the copyright holder. To view a copy of this licence, visit http://creativecommons.org/licenses/by/4.0/. The Creative Commons Public Domain Dedication waiver (http://creativeco mmons.org/publicdomain/zero/1.0/) applies to the data made available in this article, unless otherwise stated in a credit line to the data. 
Study using DNA methylation and gene expression data from brain tissues as neuropathological staging (Braak score) advances.

Conclusions: Our results confirm that PM20D1 is an $\mathrm{mQTL}$ in $\mathrm{AD}$ and demonstrate that it plays a dynamic role at different stages of the disease. Further in-depth study is thus warranted to fully decipher its role in the evolution of AD and potentially explore its utility as a blood-based biomarker for AD.

Keywords: Alzheimer's disease, Epigenetics, PM20D1, Mixed-effects model

\section{Background}

Over a decade of genetics research on Alzheimer's disease (AD) has identified $30+$ susceptibility genes which altogether account for less than $50 \%$ of the heritability of late-onset Alzheimer's disease (LOAD) [1]. With continuous efforts to thoroughly characterize the genetic risk for LOAD, emerging evidence suggests that epigenetics also plays a significant role in disease pathogenesis, progression and resilience [2-4]. Among the various epigenetic modifications, DNA methylation is the most widely studied mechanism due to its interpretable relationship to disease-associated gene expression, the availability of different experimental assays and advanced analysis tools, thus enabling high throughput processing. It has also attracted increasing attention as a potential biomarker, with accumulating evidence indicating that abnormal methylation can be used for detection and diagnosis of disease, prediction of response to therapeutic interventions and prognosis of outcome [5]. For AD, the methylation profile from peripheral tissues such as blood would be especially useful as a diagnostic tool due to the noninvasive, easily measurable characteristics and the possibility of longitudinal study [6].

Specifically for LOAD, in postmortem brain tissues, cell type-specific methylation signatures and differential methylation dynamics were reported for several brain pathology-related genes such as ankyrin 1 (ANK1) [7, 8], histone deacetylases (HDACs) [9, 10] and homeobox genes (HOXs) [11, 12]. More recently, analysis from biologically and technically independent datasets focusing on the comparison between samples from healthy controls and patients with advanced-stage AD shows that only one gene, peptidase M20-domain-containing protein 1(PM20D1), a biosynthetic enzyme for a class of $\mathrm{N}$-lipidated amino acids in vivo, consistently displayed promoter hypermethylation in patients with AD [13]. Furthermore, it has been demonstrated that PM20D1 is a methylation and expression QTL coupled to an AD-risk associated haplotype defined by rs708727 and rs960603, which displays enhancer-like characteristics and contacts the PM20D1 promoter via a haplotype-dependent, CCCTC-binding-factor-mediated chromatin loop [13]. Nevertheless, these findings, which were all observed in various brain tissues, are not necessarily translatable to the whole blood due to the highly dynamic nature of peripheral tissues and the heterogeneity of disease. Multiple studies have attempted to reproduce the observations of methylation changes in brain and peripheral blood, but few consistent results have emerged. For example, hypermethylation of ALK1 in two cortical regions (superior temporal gyrus and prefrontal cortex) of AD subjects has been observed; however, these alterations were not observed in whole blood obtained premortem from the same individuals [8]. TNF- $\alpha$ shows significant hypomethylation in the cortex samples of $\mathrm{AD}$ patients but not in their blood samples [14]. These findings indicate that some of the epigenetic mechanisms being uncovered in AD pathology are only relevant to brain cells, not blood cells. Furthermore, many epigenetic alterations observed in blood cells could not be detected in the brain [15]. A global correlation of epigenetic changes in the brain with peripheral tissues is yet to be established.

Still there is evidence indicating that blood DNA methylation dynamics may mediate detectable transcriptomic changes [8] and many DNA methylation variations have consistent effects across tissues [16]. Analysis of methylomic co-variation between tissues, specifically between whole blood and different regions of brain demonstrated that for a portion of the methylation sites, blood methylation levels are correlated with those from brain, and suggested the utility of using a blood-based approach to identify potential biomarkers of psychiatric disease phenotypes [17]. Given the difficulty in accessing and collecting brain tissue samples especially longitudinally to track disease diagnosis and progression, valuable information could still be obtained from blood-based DNA methylation studies [6].

We set out to utilize the data available from the ADNI study which comprised a large cohort with both bloodbased longitudinal DNA methylation data and crosssectional gene expression data, by starting from baseline DNA methylation measurements in the stable diagnostic groups to detect the differential methylated regions (DMRs) at the group level, then tracking the dynamic change of the DMR among the different diagnostic subgroups, including those converters. It is one of the ongoing efforts to exploit the data to seek understanding of the heterogenicity and dynamic nature of AD. The ample 
information possessed by the longitudinal data in the ADNI cohort could help validate the previous findings observed in brain tissues while providing unprecedented perspectives into the dynamics of the epigenetic background of $\mathrm{AD}$. Based on the ADNI data, we now report the preliminary findings that are observed at PM20D1 locus: (1) confirmation of PM20D1 as a methylation and expression QTL coupled to an AD-risk associated genotype defined by rs708727 in peripheral blood, mostly consistent with what is been observed in the brain tissues from the hippocampus and the frontal cortex; and (2) the methylation profile's moving direction at the early stage of $\mathrm{AD}$, which is contrary to what is observed in brain tissues with advanced-stage AD (Braak staging $\geq 5$ ) [13]. We modeled the alteration of methylation levels at the promoter regions of the gene as a function of disease progression and validated the findings in a separate cohort with both methylation and gene expression data from brain tissues. This work helps characterize a comprehensive picture for the epigenetic change at the PM20D1 gene locus associated with the onset, as well as progression of $\mathrm{AD}$ and suggests its potential as a blood-based biomarker.

\section{Results}

\section{Methylation data processing and quality check (QC)}

The details of the ADNI study, the experiment design for methylation array and initial data QC are available online at: http://adni.loni.usc.edu/methods/documents/ and http://adni.loni.usc.edu/data-samples/access-data/. Briefly, DNA was isolated and plated out at NCRAD and DNA methylation profiling was performed at AbbVie for a total of 1920 samples, including 1719 unique samples and 201 technical replicates (653 unique individuals). Longitudinal DNA samples at baseline, and +up to 4 years, sampled at $\sim 1$ year apart were obtained from all subjects. Samples were randomized using a modified incomplete balanced block design, whereby all samples from a subject were placed on the same chip, with remaining chip space occupied by age- and sex-matched samples. Subjects from different diagnosis groups were placed on the same chip to avoid confounding. Unused chip space was leveraged for technical reproducibility assessment via replicated DNA samples. Sample and probe quality control, including detection $\mathrm{P}$ values, checking the sex of samples and sample identity, removed 15 samples from the data. The released raw data from ADNI methylation array consist of 1905 samples at >850,000 CpG sites, creating unique challenges in data processing and QC. We chose to process the data with the most recently developed 'bigmelon' package in R, a memory efficient method to import the raw data and export the data matrices without any filtering steps. All the downstream analyses were accomplished by the ChAMP pipeline for an integrated workflow, which employs a set of tools for filtering, normalization, batch correction and cell type correction for data from whole blood samples. Preprocessing of the data resulted in 685,446 probes for 1,905 samples. Singular value decomposition (SVD) analysis [18] after batch correction found no covariates contribute to significant components of variation. Mean value for each estimated cell proportion shows the cell type profile agrees well with known cell type profile for whole blood samples, with granulocytes/neutrophils making up $50-60 \%$ of the samples and lymphocytes/monocytes making up the rest (Fig. 1). At baseline, the proportion of granulocytes was higher $(p=0.0027, \mathrm{fdr}=0.048$, two-sided Student's $t$ test), and that of CD8T cells was lower $(p=0.0059$, $\mathrm{fdr}=0.053$, two-sided Student's $t$ test) in the AD patients, indicating an alteration of immune response in the $A D$ patients. All other cell types remain comparable among the subjects. This observation also does not change in the full longitudinal data time frame.

\section{Differentially methylated regions (DMRs) at baseline}

We first focused on those subjects (579 out of 649) whose age $>65$ at the baseline measurements (LOAD). A subset of the subjects (424) kept a stable diagnosis during the whole sampling time frame throughout all the visits; thus, their baseline measurements were used for DMR detection. A region of $\sim 900 \mathrm{bp}$ on chromosome $1 \mathrm{dem}-$ onstrated hypomethylation in the AD patients in comparison with control $(p=7.48 \mathrm{E}-06$, fwer $=0.004)$, as well as $\mathrm{AD}$ vs $\mathrm{MCI}(p=9.11 \mathrm{E}-05$, fwer $=0.048)$. This is also the only DMR passing the corrected fwer cutoff (0.05). This region also demonstrated moderate hypomethylation when we compared MCI against control $(p=0.00237$, fwer $=0.748)$, indicating a gradual methylation decrease during disease progression (Table 1, Fig. 2, Additional file 1: Table S1). Interestingly, this region lies within the promoter region of gene PM20D1, the recently reported $\mathrm{mQTL} / \mathrm{eQTL}$ for AD, albeit it is hypermethylated in some brain regions of subjects with advanced LOAD [13]. We therefore focused on the longitudinal data for the $10 \mathrm{CpG}$ probes from the Illumina EPIC array in this region (Table 2) to fully dissect the methylomic changes for PM20D1 in peripheral blood, throughout the course of disease progression.

\section{Allelic dosage effect in the DMR}

The effects on the methylation values $\left(\beta=\frac{M}{M+U+\alpha}\right.$, where $M$ and $U$ are the methylated and unmethylated signal intensities, and $\alpha$ is an offset) in the DMR regions from alternate allele doses of the two SNP sites were quantitatively evaluated by linear regression at the baseline. We stratified the cohort by diagnostic status during 


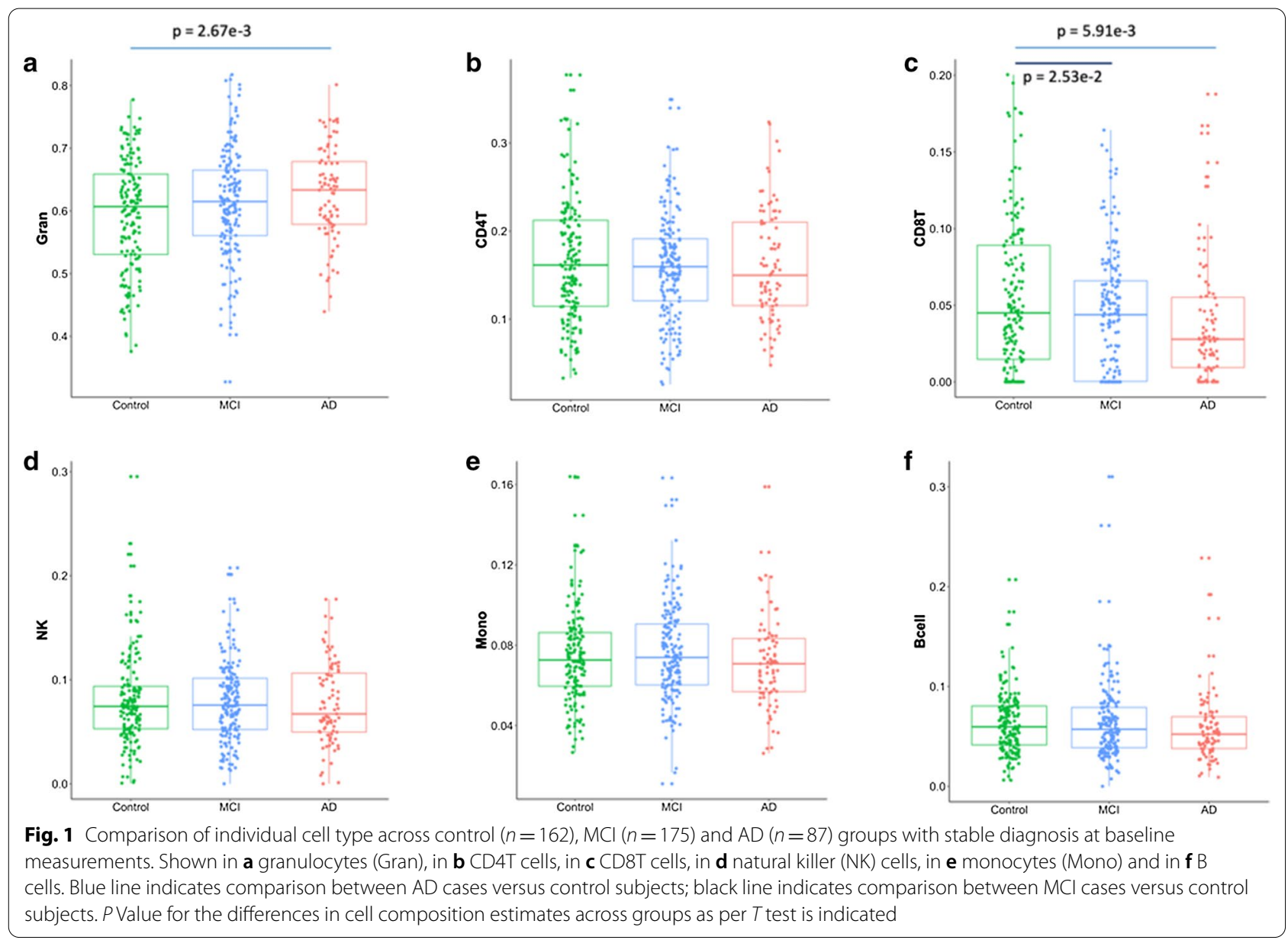

Table 1 The differentially methylated regions (DMRs) in PM20D1 promoter region as detected by the comparisons among control, $\mathrm{MCl}$ and $\mathrm{AD}$

\begin{tabular}{|c|c|c|c|c|c|c|c|c|c|c|c|}
\hline Comparison & Rank & Chr & Start & End & Width & Value & Area & $p$ value & fwer & $\begin{array}{l}\text { p.value } \\
\text { Area }\end{array}$ & Fwer area \\
\hline$A D$ versus control & 1 & chr1 & $205,818,668$ & $205,819,609$ & 941 & -0.476 & 5.23 & $7.48 \mathrm{E}-06$ & 0.004 & 0.000217 & 0.112 \\
\hline$A D$ versus $M C l$ & 1 & chr1 & $205,818,956$ & $205,819,609$ & 653 & -0.373 & 3.73 & $9.11 \mathrm{E}-05$ & 0.048 & 0.000615 & 0.276 \\
\hline $\mathrm{MCl}$ versus control & 5 & chr1 & $205,818,956$ & $205,819,609$ & 653 & -0.143 & 1.43 & 0.00237 & 0.748 & 0.00597 & 0.952 \\
\hline
\end{tabular}

the full sampling time frame by AD only (87 subjects), MCI only (174 subjects), control only (162 subjects) and converters (117 subjects), and effect was evaluated for the three stable diagnosis groups, respectively. We observed that in all diagnostic groups, rs708727 had the most prominent effect as expected $(p<2 \mathrm{e}-16$ in control and MCI, $p<0.0001$ in AD), while rs960603 was not significant for any of the 3 groups $(p>0.1)$ (Table 3, Fig. 3, Additional file 2: Table S2). The slopes of rs708727 were comparable in all diagnostic groups $(p>0.05$ by testing the differences of the slopes). For both control and MCI groups, age and sex were not significantly associated $(p>0.05)$, while in $\mathrm{AD}$, sex plays a role in the methylation change $(p<0.05$ in 6 probes, $p<0.1$ in 9 probes in total), where female shows higher $\beta$ values.

\section{Longitudinal data analysis}

To evaluate longitudinal changes in methylation levels, we first stratified the cohort by diagnosis status.For stable diagnosis groups ( $\mathrm{AD} / \mathrm{MCI} / \mathrm{control}), \beta$ values were fit to age, with sex, allelic dosages for rs708727 and rs960603 as fixed effect covariates by LME models. Consistent with the baseline data, allelic dose for rs708727 demonstrates the most dominant effect on the methylation levels in all 


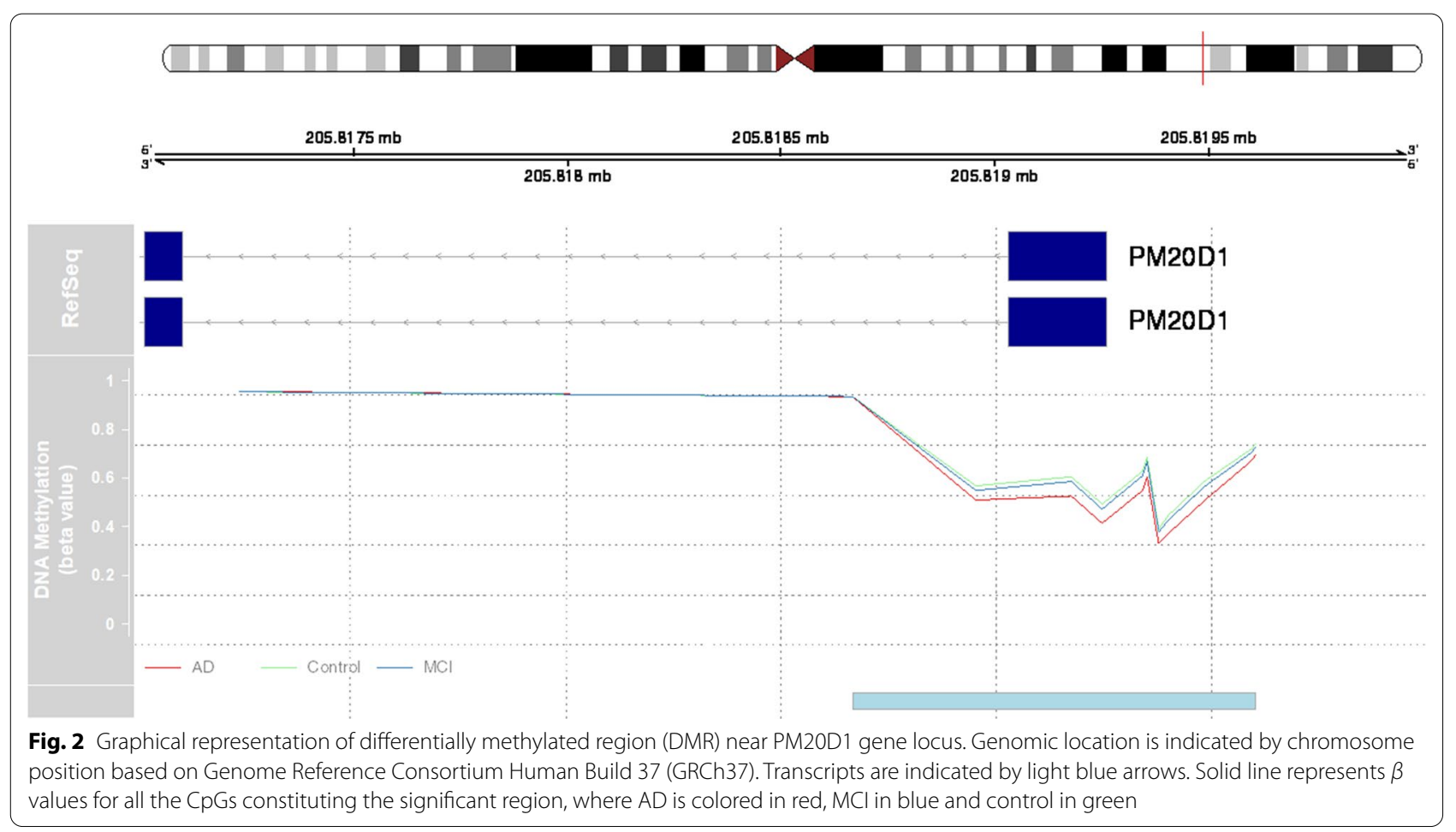

Table 2. 10 CpG probes and their respective $\beta$ values from EPIC array in the DMR region at PM20D1

\begin{tabular}{llllll}
\hline CpG probes & Control & MCI & AD & MAPINFO & UCSC refgene group \\
\hline cg17178900 & $0.568 \pm 0.200$ & $0.549 \pm 0.193$ & $0.509 \pm 0.208$ & 205818956 & Body \\
cg14159672 & $0.605 \pm 0.233$ & $0.586 \pm 0.225$ & $0.526 \pm 0.241$ & 205819179 & 1 st exon \\
cg14893161 & $0.493 \pm 0.220$ & $0.472 \pm 0.217$ & $0.414 \pm 0.216$ & 205819251 & 5 'UTR; 1 st exon \\
cg07533224 & $0.627 \pm 0.221$ & $0.609 \pm 0.215$ & $0.549 \pm 0.232$ & 205819345 & TSS200 \\
cg12898220 & $0.685 \pm 0.207$ & $0.667 \pm 0.201$ & $0.605 \pm 0.224$ & 205819356 & TSS200 \\
cg05841700 & $0.390 \pm 0.190$ & $0.378 \pm 0.182$ & $0.330 \pm 0.177$ & 205819383 & TSS200 \\
cg11965913 & $0.447 \pm 0.240$ & $0.427 \pm 0.234$ & $0.373 \pm 0.229$ & 205819406 & TSS200 \\
cg24503407 & $0.589 \pm 0.213$ & $0.568 \pm 0.210$ & $0.510 \pm 0.220$ & 205819492 & TSS1500 \\
cg16334093 & $0.720 \pm 0.136$ & $0.705 \pm 0.210$ & $0.672 \pm 0.147$ & 205819600 & TSS1500 \\
cg07157834 & $0.739 \pm 0.127$ & $0.727 \pm 0.129$ & $0.695 \pm 0.136$ & 205819609 & TSS1500 \\
\hline
\end{tabular}

the groups $(p<2.2 \mathrm{e}-16)$, while that of rs960603 is not significant $(p>0.1)$. On top of the effect of allelic dose of rs708727, it also shows an increasing trend of the slope from control to $\mathrm{MCI}$, then to $\mathrm{AD}$ (AD vs control, $p<0.1$ at 4 probes). For both MCI and control groups, no other variables show any effects on any of the probes $(p>0.1)$. In contrast, in $\mathrm{AD}$ patients, after controlling allelic dose effect, at least half of the probes (4 probes with $p<0.05,6$ probes with $p<0.1$ ) still show significant age-dependent methylation elevations (Table 4, Fig. 4a-c, Additional file 3: Table S3). Also consistent with the model of allelic dosage effect at baseline, a majority of the probes (6 probes with $p<0.05,9$ probes with $p<0.1$ ) also show sexdependent methylation elevation. Another interesting finding is the weak positive correlation between methylation level and p-tau181 values in plasma (3 probes with $p<0.05$, Additional file 3: Table S3) as well as the sex effect ( 3 probes with $p<0.05,6$ probes with $p<0.1$ ).

To test if there is a turning point for the methylation level of PM20D1 in the disease progression process, we employed the U-shape test by the two-lines method on the longitudinal data for the converters group (117 subjects). We adopted the 'two-lines' U-shape test approach to demonstrate (and statistically test) that there is a 
Table 3 Model summary for the allelic dosage effects of rs708727 on the $\beta$ values at one of the representative CpG probes (cg14159672)

\begin{tabular}{|c|c|c|c|c|c|c|c|c|}
\hline \multirow[t]{2}{*}{$d x$} & \multicolumn{6}{|l|}{ Effects } & \multicolumn{2}{|c|}{ Model statistics } \\
\hline & variant & Estimate & SE & $t$ value & $\operatorname{Pr}(>|t|)$ & Signif. codes & & \\
\hline \multirow[t]{5}{*}{ Control } & (Intercept) & 0.1745 & 0.1522 & 1.15 & 0.254 & & Residual SD & 0.1444 \\
\hline & rs708727 & 0.2662 & 0.0218 & 12.23 & $<2 \mathrm{E}-16$ & $* * *$ & Multiple $r^{2}$ & 0.6267 \\
\hline & rs960603 & -0.0120 & 0.0229 & -0.52 & 0.602 & & Adjusted $r^{2}$ & 0.6172 \\
\hline & Age & 0.0031 & 0.0019 & 1.66 & 0.100 & & F-statistic & 65.9 (4/157 DF) \\
\hline & Sex & -0.0165 & 0.0230 & -0.72 & 0.476 & & $p$ value & $<2.2 \mathrm{E}-16$ \\
\hline \multirow[t]{5}{*}{$\mathrm{MCl}$} & (Intercept) & 0.3613 & 0.1437 & 2.51 & 0.013 & * & Residual SD & 0.1532 \\
\hline & rs708727 & 0.2544 & 0.0228 & 11.17 & $<2 \mathrm{E}-16$ & $* * *$ & Multiple $r^{2}$ & 0.5468 \\
\hline & rs960603 & -0.0125 & 0.0226 & -0.55 & 0.580 & & Adjusted $r^{2}$ & 0.536 \\
\hline & Age & 0.0001 & 0.0019 & 0.03 & 0.973 & & F-statistic & 50.97 (4/169 DF) \\
\hline & Sex & 0.0030 & 0.0239 & 0.13 & 0.901 & & $p$ value & $<2.2 \mathrm{E}-16$ \\
\hline \multirow[t]{5}{*}{$A D$} & (Intercept) & -0.0555 & 0.2317 & -0.24 & 0.811 & & Residual SD & 0.173 \\
\hline & rs708727 & 0.2654 & 0.0455 & 5.84 & $1.02 \mathrm{E}-07$ & $* * *$ & Multiple $r^{2}$ & 0.5096 \\
\hline & rs960603 & 0.0016 & 0.0394 & 0.04 & 0.968 & & Adjusted $r^{2}$ & 0.4857 \\
\hline & Age & 0.0036 & 0.0029 & 1.27 & 0.208 & & F-statistic & 21.31 (4/82 DF) \\
\hline & Sex & 0.0874 & 0.0400 & 2.19 & 0.032 & * & $p$ value & $4.49 E-12$ \\
\hline \multirow[t]{5}{*}{ All stable } & (Intercept) & 0.1951 & 0.0941 & 2.07 & 0.039 & * & Residual SD & 0.155 \\
\hline & rs708727 & 0.2643 & 0.0150 & 17.58 & $<2 \mathrm{e}-16$ & $* * *$ & Multiple $r^{2}$ & 0.5638 \\
\hline & rs960603 & -0.0090 & 0.0150 & -0.60 & 0.549 & & Adjusted $r^{2}$ & 0.5597 \\
\hline & Age & 0.0020 & 0.0012 & 1.69 & 0.093 & & F-statistic & 135.1 (4/418 DF) \\
\hline & Sex & 0.0143 & 0.0152 & 0.94 & 0.348 & & $p$ value & $<2.2 \mathrm{E}-16$ \\
\hline
\end{tabular}

Signif. codes: $0^{\prime * * * \prime} 0.001^{* * * \prime} 0.01^{* * \prime} 0.05 " 0.1 " 1$
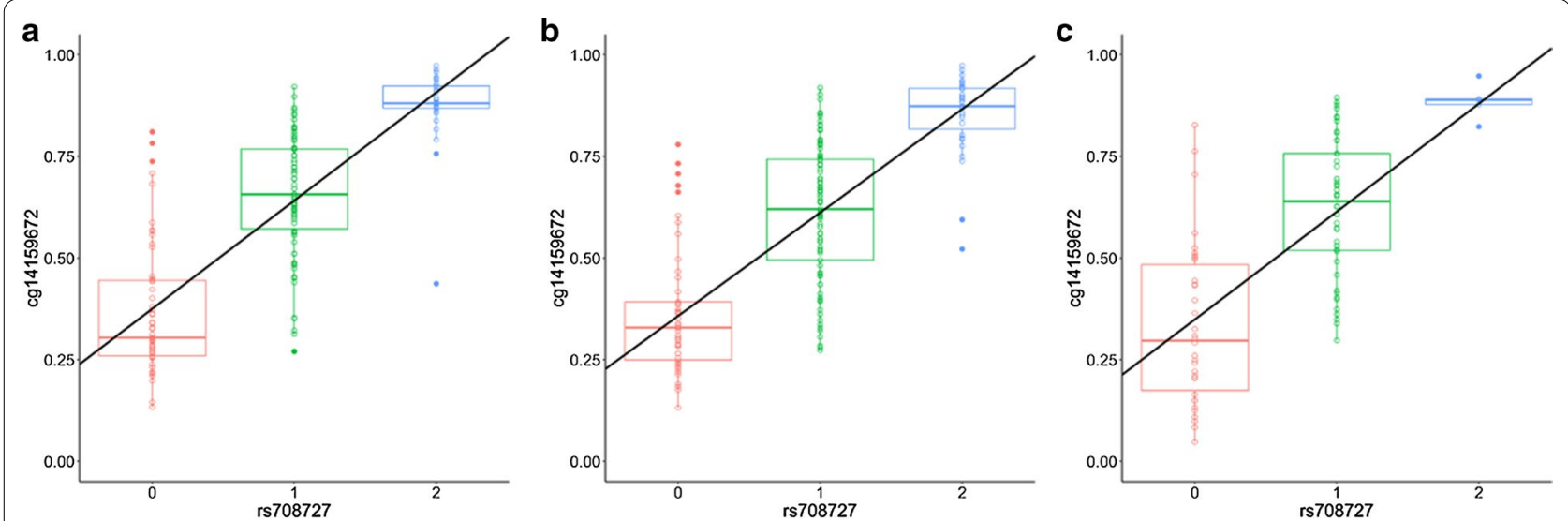

Fig. 3 Methylation change as allelic dose of rs 708727 changes modeled by $\beta$ values at baseline regressed with allelic dose of rs708727 at one of the representative CpG probes (cg14159672). Scatter plot is colored by the allelic doses of rs708727 where red $=0$ (GG), green $=1$ (GA), and blue $=2$ (AA). An overall linear fit line is also shown. Panel $\mathbf{a}$ depicts control group, $\mathbf{b}$ depicts $\mathrm{MCl}$ group, and $\mathbf{c}$ depicts AD group

non-monotonical trajectory in the methylation level as a function of disease progression, by characterizing the nonlinearity without making functional-form assumptions about $f(x)$. This approach has also been adopted in the AD research field previously to model disease progression [19]. For each probe, a clear U shape was observed ( $p<0.01$ on both slopes), with the breakpoint identified at $\sim 78$ to 79 years of old (Additional file 3: Table S3, Fig. 4d). Allelic doses of rs708727 and rs960603 also show significant effect on the correlation, although the former is more prominent than the later. Consistent with our previous analysis, we also detected a sex effect 
Table 4 Model summary for the LME modeling between age and the $\beta$ values at one of the representative CpG probes (cg14893161). Fixed effects from age, sex, allelic dosages of the two SNPs and random effects from subject (RID), chip slide and array are shown

\begin{tabular}{|c|c|c|c|c|c|c|c|c|c|c|c|}
\hline \multirow[t]{2}{*}{$d x$} & \multicolumn{7}{|c|}{ Fixed effects } & \multicolumn{4}{|c|}{ Random effects } \\
\hline & Variant & Estimate & SE & $d f$ & $t$ value & $\operatorname{Pr}(>|t|)$ & Signif. codes & Groups & Name & Variance & SD \\
\hline \multirow[t]{5}{*}{ Control } & (Intercept) & 0.2544 & 0.1185 & 312.70 & 2.15 & 0.0326 & * & RID & (Intercept) & 0.0154 & 0.1239 \\
\hline & Age & 0.0010 & 0.0014 & 340.10 & 0.68 & 0.4948 & & Slide & (Intercept) & 0.0030 & 0.0544 \\
\hline & Sex & -0.0299 & 0.0218 & 158.80 & -1.37 & 0.1715 & & Array & (Intercept) & 0.0000 & 0.0025 \\
\hline & rs708727 & 0.2392 & 0.0203 & 154.90 & 11.76 & $<2 \mathrm{E}-16$ & $* * *$ & Residual & & 0.0017 & 0.0408 \\
\hline & rs960603 & 0.0018 & 0.0215 & 159.60 & 0.08 & 0.9339 & & & & & \\
\hline \multirow[t]{5}{*}{$\mathrm{MCl}$} & (Intercept) & 0.2973 & 0.1064 & 351.20 & 2.795 & 0.00547 & $* *$ & RID & (Intercept) & 0.0123 & 0.1108 \\
\hline & Age & -0.0010 & 0.0013 & 385.40 & -0.710 & 0.4782 & & Slide & (Intercept) & 0.0089 & 0.0943 \\
\hline & Sex & 0.0071 & 0.0221 & 168.30 & 0.321 & 0.7482 & & Array & (Intercept) & 0.0001 & 0.0077 \\
\hline & rs708727 & 0.2676 & 0.0210 & 167.00 & 12.742 & $<2 \mathrm{E}-16$ & $* * *$ & Residual & & 0.0014 & 0.0374 \\
\hline & rs960603 & -0.0038 & 0.0206 & 165.30 & -0.187 & 0.8520 & & & & & \\
\hline \multirow[t]{5}{*}{$A D$} & (Intercept) & -0.2992 & 0.1659 & 117.40 & -1.803 & 0.07391 & & RID & (Intercept) & 0.0156 & 0.1250 \\
\hline & Age & 0.0056 & 0.0020 & 122.80 & 2.775 & 0.00638 & $* *$ & Slide & (Intercept) & 0.0081 & 0.0899 \\
\hline & Sex & 0.0696 & 0.0351 & 83.48 & 1.983 & 0.0506 & & Array & (Intercept) & 0.0000 & 0.0050 \\
\hline & rs708727 & 0.2722 & 0.0379 & 69.96 & 7.179 & $5.90 \mathrm{E}-10$ & $* * *$ & Residual & & 0.0019 & 0.0432 \\
\hline & rs960603 & 0.0006 & 0.0335 & 77.22 & 0.019 & 0.9849 & & & & & \\
\hline \multirow[t]{5}{*}{ All stable } & (Intercept) & 0.0996 & 0.0715 & 798.00 & 1.391 & 0.1645 & & RID & (Intercept) & 0.0132 & 0.1150 \\
\hline & Age & 0.0015 & 0.0009 & 864.10 & 1.733 & 0.0835 & & Slide & (Intercept) & 0.0080 & 0.0897 \\
\hline & Sex & 0.0089 & 0.0144 & 442.00 & 0.622 & 0.5343 & & Array & (Intercept) & 0.0000 & 0.0054 \\
\hline & rs708727 & 0.2831 & 0.0128 & 340.10 & 22.051 & $<2 \mathrm{e}-16$ & $* * *$ & Residual & & 0.0017 & 0.0408 \\
\hline & rs960603 & 0.0038 & 0.0131 & 374.50 & 0.288 & 0.7733 & & & & & \\
\hline
\end{tabular}

Signif. codes: 0 ***'0.001 ${ }^{* * \prime} 0.01^{* \prime} 0.05$ ". $0.1^{\prime \prime 1}$

in the majority of the probes ( 5 probes with $p<0.05,7$ probes with $p<0.1, t$ test).

\section{Correlation of methylation data with gene expression}

To characterize associations between methylation changes and expression of PMD20D1, we integrated available DNA methylation data from subjects with a stable diagnosis, with matched whole blood gene expression data. For all 10 probes, we observed an inverse correlation between probe methylation $\beta$ value and PM20D1 expression (Additional file 4: Table S4, Fig. 5), with $p<0.001$ at 6 probes and $p<0.01$ at 8 probes. We again observed a significant association between dosage of rs708727 and PM20D1 expression $(p<0.0001, t$ test), while that of rs960603 shows negligible effect $(p>0.1, \mathrm{t}$ test). We did not observe associations between sex, age or diagnosis and PM20D1 gene expression.

Strikingly, we observed that the observed association between cg05841700 and PM20D1 was entirely driven by subjects with an allelic dose of 0, homozygous GG at rs708727. When the correlation is stratified by allelic doses of rs708727, it is apparent that only in the hypomethylated samples (allelic dose $=0$, homozygous GG) there is a significant linear correlation (Fig. 5, red line and dots). There is no such linear correlation in the heterozygous samples (allelic dose $=1$, heterozygous GA, Fig. 5, green line and dots), or even less so in the hypermethylated samples (allelic dose $=2$, homozygous AA, Fig. 5, blue line and dots). This is consistent with the recent discovery that the methylation level of PM20D1 promoter cannot be directly translated into gene expression, due to blockage of an enhancer downstream for the hypermethylated groups (AD-risk associated haplotype). Only from individuals with unmethylated PM20D1 where the enhancer region physically interacts with the promoter, can PM20D1 transcription begin [13]. The current study provides numeric representations of the relationship and confirms a similar observation that is made in a peripheral tissue (blood) in comparison with the findings originally reported in brain tissue samples [13].

\section{Data from brain tissue support findings in whole blood}

As a validation of the methylation alteration at the PM20D1 promoter region observed in blood-based data, we investigated DNA methylation changes at $6 \mathrm{CpG}$ sites profiled from postmortem brain prefrontal cortex tissue samples collected as part of the ROSMAP cohort. We observed a strong association between CpG methylation 

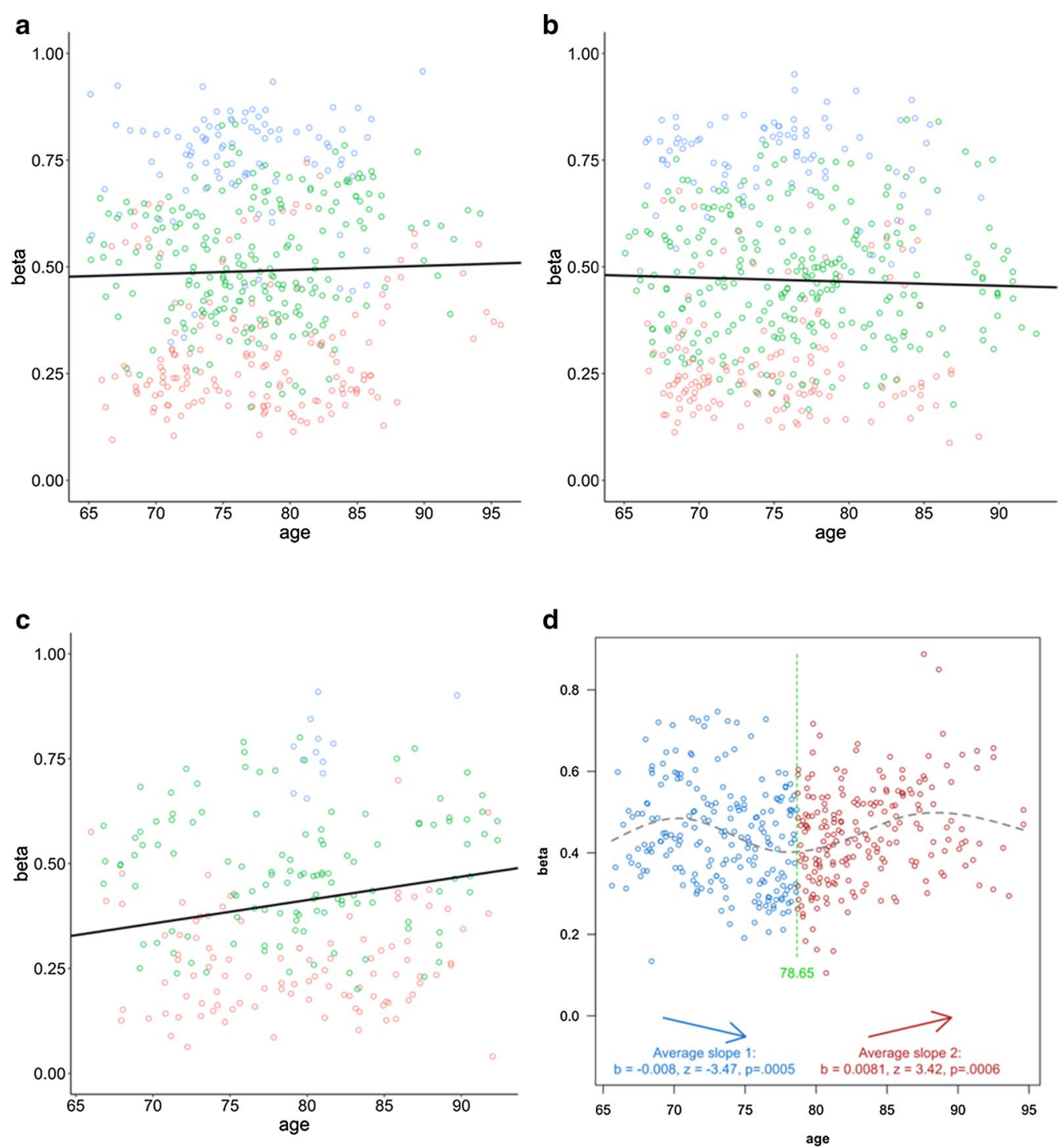

Fig. 4 Methylation change as disease/age progresses modeled by $\beta$ values regressed with age at one of the representative $C p G$ probes (cg14893161). Scatter plot is colored by the allelic doses of rs 708727 where red $=0$ (GG), green =1 (GA), and blue $=2$ (AA) in panels a-c. An overall linear fit line is also shown. Panel $\mathbf{a}$ depicts control group, $\mathbf{b}$ depicts $\mathrm{MCl}$ group, and $\mathbf{c}$ depicts AD group. Panel $\mathbf{d}$ depicts all the conversion cases where a U-shape is fit and a break point is marked

and Braak staging $(p<0.05)$ in participants with an AD diagnosis (Table 5, Additional file 5: Table S5, Fig. 6). We did not observe any association in the control group for any of the $8 \mathrm{CpG}$ sites, and only one probe was found to be significantly associated with Braak staging in the MCI group ( $p=0.038$ at cg24503407), and another marginally significant $(p=0.070$ at $\operatorname{cg} 17178900)$. When combined together, there is a linear correlation $(p<0.05)$ between methylation level and Braak score at 6 out of the 8 probes for all the subjects. This correlation could be attributed to the larger sample size, AD subgroup and wider range of Braak stages in the overall group. Again, rs708727 was found to be a highly significant $(p<2 \mathrm{e}-16)$ covariant of all probes, while rs960603 is not. In contrast with our previous analysis, we did not observe an association with sex. Interestingly, the correlation between DNA methylation levels and pathological biomarkers (amyloid and tangles) shows a positive association of quantitative overall amyloid level at 3 probes in the AD subgroup, 1 probe in the MCI subgroup and 0 in the controls out of the 8 probes in total. For tangles, there is no association of PM20D1 methylation at all for any of the diagnostic subgroups, but a weak association in the overall group $(p<0.05$ for 4 out of 8 probes, Additional file 5: Table S5). 


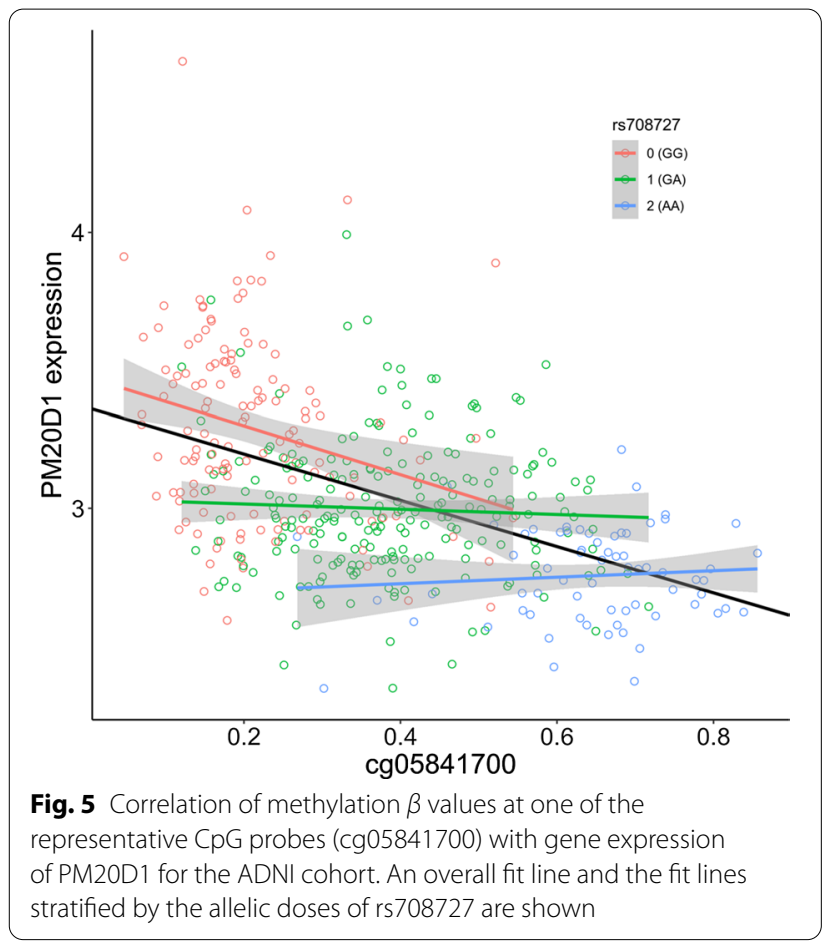

We also observed an inverse linear correlation between $\beta$ value and PM20D1 expression (Additional file 6: Table S6, Additional file 9: Fig. S1). Consistent with our findings within the blood-based ADNI data, after stratification by rs708727 genotype, the observed linear correlation was primarily attributed to the lower AD-risk populations (rs708727 allelic dose =0, GG).

\section{Direction comparison of DNA methylation between brain tissues and whole blood}

The consistent findings between the different brain regions and the peripheral blood from different cohorts prompted a direct comparison between DNA methylation in blood with brain regions for the $\mathrm{CpG}$ probes at PM20D1 promoter region, using published results in the third independent cohort (the London cohort). Across the $8 \mathrm{CpG}$ probes from the Methylation $450 \mathrm{~K}$ array at PM20D1 promoter region, the positive correlation of methylation between blood and any of the four brain regions is all highly significant, with correlation coefficient ranging from 0.857 to $0.976(p<0.0001$, Additional file 7: Table S7, Additional file 9: Fig. S2). Of note, this correlation is regardless of age, sex or pathological state of the individuals, highlighting a consistent interindividual covariance in whole blood and that observed in all four brain regions, specific to the promoter region of PM20D1. The clear trimodal distribution of DNA methylation suggests that the mQTL mediates much of the observed cross-tissue similarities, and the profile at PM20D1 in blood could be used as a proxy to predict DNA methylation levels in the brain [20].

In summary, our results collectively demonstrate that there is a U-shaped dynamic trajectory in the methylation profile for PM20D1 promoter in the whole blood samples from ADNI cohort, from normal state to MCI then to LOAD. The change trend of the methylation profile at the same promoter in the brain samples from the ROSMAP cohort, and the remarkable correlation between blood and brain and between several brain tissues in the third cohort confirm that this is a universal observation conserved in different tissue types across different demographics.

\section{Discussions}

Early diagnosis and treatment of AD have been called out as the most urgent problem to tackle for this second century disease [21]. Nevertheless, to date not a single easyto-measure biomarker has been fully established for its diagnosis and it remains elusive to identify blood-based biomarkers that will pinpoint $\mathrm{AD}$ in its earliest phases. As the most important goal is to detect $\mathrm{AD}$ at the earliest possible stage (pre-dementia) and identify ways to track the disease's progression with biomarkers, ADNI data provide unique opportunity to observe the drastic change of molecular profiles during disease progression. Data obtained from microarray whole-genome DNA methylation profiling enable large scope in both time frame and sample size offering unprecedented insights into the dynamics of epigenetic signatures during the early phases in a large AD cohort. On the other hand, epigenetic signatures are largely tissue-specific; so understanding how observations obtained from peripheral tissue such as blood might relate to brain tissues needs further examination.

As a pioneering study in exploring the whole dataset, we made a direct comparison of baseline measurements among control, MCI, and AD groups from the wholegenome methylation profiling data. We found that the promoter of PM20D1 gene locus consistently displayed hypomethylation from control to $\mathrm{MCI}$, and even further to symptomatic AD. PM20D1 has recently been reported as an mQTL in two major $\mathrm{AD}$ affected brain regions, the hippocampus and the frontal cortex, based on the comparisons between samples from healthy controls and patients with advanced-stage AD, although it is been found to be hypermethylated in the latter [13]. In the report, an allele-dependent correlation with PM20D1 promoter methylation is identified for the rs708727rs960603 haplotype. In the proposed mechanistic model, PM20D1 has been suggested as a protective gene of $\mathrm{AD}$, whose elevated expression levels might provide a 
Table 5 Model summary for the effects of Braak staging on the $\beta$ values at one of the representative CpG probes (cg26354017)

\begin{tabular}{|c|c|c|c|c|c|c|c|c|}
\hline \multirow[t]{2}{*}{$d x$} & \multicolumn{6}{|l|}{ Effects } & \multirow{2}{*}{\multicolumn{2}{|c|}{ Model statistics }} \\
\hline & variant & Estimate & SE & $t$ value & $\operatorname{Pr}(>|t|)$ & Signif. codes & & \\
\hline \multirow[t]{6}{*}{ Control } & (Intercept) & 0.3302 & 0.1194 & 2.767 & 0.006 & $* *$ & Residual SD & 0.1008 \\
\hline & Braak & 0.0039 & 0.0064 & 0.602 & 0.548 & & Multiple $r^{2}$ & 0.8457 \\
\hline & rs708727 & 0.3340 & 0.0143 & 23.379 & $<2 \mathrm{e}-16$ & $* * *$ & Adjusted $r^{2}$ & 0.8419 \\
\hline & rs960603 & 0.0095 & 0.0140 & 0.682 & 0.496 & & F-statistic & 226.9 (5/207 DF) \\
\hline & msex & 0.0043 & 0.0147 & 0.290 & 0.772 & & $p$ value & $<2.2 \mathrm{E}-16$ \\
\hline & age_death & -0.0016 & 0.0014 & -1.123 & 0.263 & & & \\
\hline \multirow[t]{6}{*}{$\mathrm{MCl}$} & (Intercept) & 0.2988 & 0.1729 & 1.73 & 0.086 & & Residual SD & 0.1016 \\
\hline & Braak & 0.0133 & 0.0082 & 1.63 & 0.106 & & Multiple $r^{2}$ & 0.8476 \\
\hline & rs708727 & 0.3446 & 0.0182 & 18.98 & $<2 \mathrm{e}-16$ & $* * *$ & Adjusted $r^{2}$ & 0.8423 \\
\hline & rs960603 & -0.0203 & 0.0183 & -1.11 & 0.269 & & F-statistic & 159.1 (5/143 DF) \\
\hline & msex & 0.0245 & 0.0175 & 1.40 & 0.164 & & $p$ value & $<2.2 \mathrm{E}-16$ \\
\hline & age_death & -0.0014 & 0.0020 & -0.70 & 0.483 & & & \\
\hline \multirow[t]{6}{*}{$A D$} & (Intercept) & 0.2564 & 0.1636 & 1.57 & 0.118 & & Residual SD & 0.092 \\
\hline & Braak & 0.0166 & 0.0056 & 2.95 & 0.004 & $* *$ & Multiple $r^{2}$ & 0.8813 \\
\hline & rs708727 & 0.3362 & 0.0146 & 23.06 & $<2 \mathrm{e}-16$ & $* * *$ & Adjusted $r^{2}$ & 0.8788 \\
\hline & rs960603 & 0.0102 & 0.0142 & 0.72 & 0.472 & & F-statistic & 351.9 (5/237 DF) \\
\hline & msex & -0.0140 & 0.0130 & -1.08 & 0.282 & & $p$ value & $<2.2 \mathrm{E}-16$ \\
\hline & age_death & -0.0013 & 0.0018 & -0.68 & 0.496 & & & \\
\hline \multirow[t]{6}{*}{ All } & (Intercept) & 0.3262 & 0.0795 & 4.10 & $4.66 \mathrm{e}-05$ & $* * *$ & Residual SD & 0.097 \\
\hline & Braak & 0.0108 & 0.0034 & 3.16 & 0.0016 & $* *$ & Multiple $r^{2}$ & 0.8590 \\
\hline & rs708727 & 0.3376 & 0.0087 & 38.79 & $<2 \mathrm{e}-16$ & $* * *$ & Adjusted $r^{2}$ & 0.8578 \\
\hline & rs960603 & 0.0038 & 0.0086 & 0.44 & 0.657 & & F-statistic & 729.9 (5/599 DF) \\
\hline & msex & 0.0015 & 0.0085 & 0.18 & 0.861 & & $p$ value & $<2.2 \mathrm{E}-16$ \\
\hline & age_death & -0.0018 & 0.0009 & -1.86 & 0.063 & & & \\
\hline
\end{tabular}

Signif. codes: $0^{\prime * * * \prime} 0.001^{\prime * * \prime} 0.01^{\prime * \prime} 0.05 " 0.1^{\prime \prime} 1$
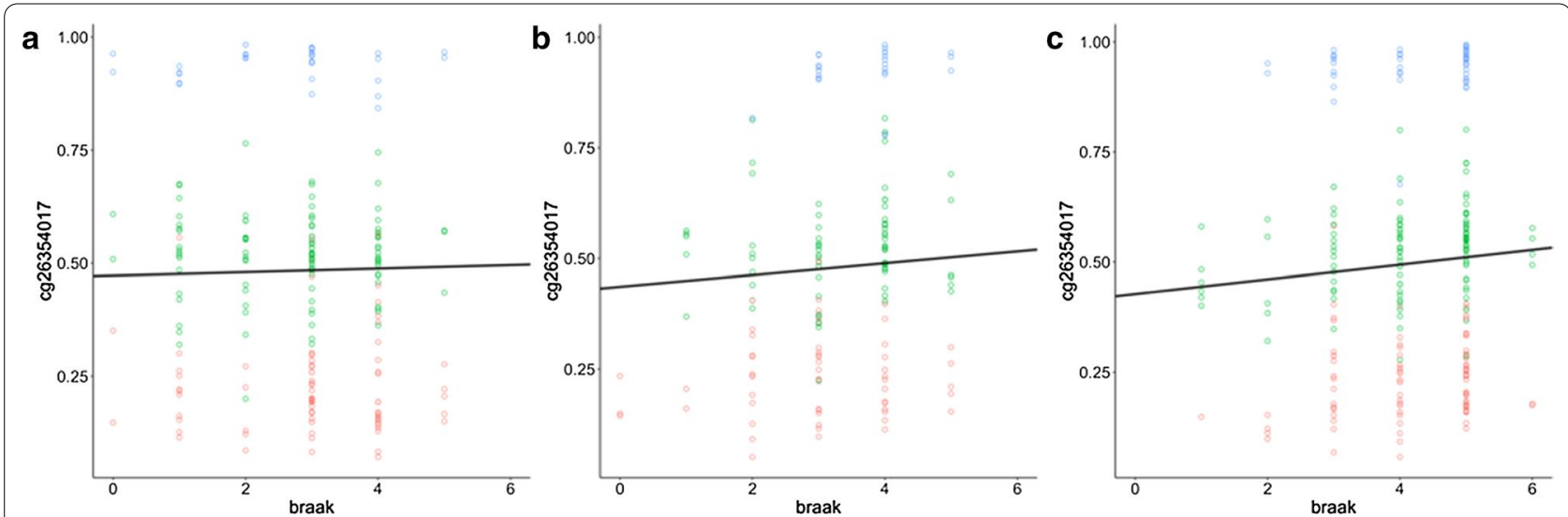

Fig. 6 Methylation change as disease progresses modeled by $\beta$ values regressed with Braak score at one of the representative CpG probes (cg26354017) from the ROSMAP brain samples. Scatter plot is colored by the allelic doses of rs 708727 where red $=0$ (GG), green $=1$ (GA), and blue $=2$ (AA). An overall linear fit line is also shown. Panel $\mathbf{a}$ depicts control group, $\mathbf{b}$ depicts $\mathrm{MCl}$ group, and $\mathbf{c}$ depicts AD group 
potential cellular defense mechanism. For AD non-riskhaplotype carriers, specifically those with homozygous reference allele haplotype at both SNP rs708727 (GG) and rs960603 (CC), PM20D1 methylation level is lower; in the presence of $\mathrm{AD}$-related stress, expression is enhanced to reduce ROS-induced cell death and $A \beta$ levels and prevent memory impairment. In contrast, in samples with hypermethylated PM20D1 (high risk, homozygous alternate allele haplotype carriers, AA/TT), the promoter region is not contacted by the enhancer and transcription does not occur, which results in low PM20D1 expression, and there is no protective effect against $A \beta$. The role of PM20D1 in AD has since then been further explored [22, 23], showing that it is the sole risk gene with consistently enriched promoter hypermethylation in AD patients, and upregulated by $A \beta$ and reactive oxygen species, and being neuroprotective when overexpressed in cell and primary cultures.

In concordance with the previous mechanistic model, we first validated the allelic dosage effects on PM20D1 promoter region in the blood samples, but found it is only due to the genotypes of rs708727. The allelic dosage of rs708727 has been found to be highly significant in determining the methylation level of the CpG sites, with higher dosage associated with higher baseline methylation quantitatively. Notably, this QTL has an effect size of $\sim 25 \%$ (Fig. 3, Table 3). Although not most common, such effect size of a QTL has been reported in multiple studies, e.g., [24, 25]. In fact, this agrees almost perfectly with what is been observed in brain tissues as reported in the aforementioned work of Sanchez-Mut et al. [13, 22]. Interestingly, although rs 960603 is reported to be co-segregated as a haplotype in nearly $85 \%$ of cases in the 1000 Genomes Project, its allelic dosage is not as significant in multiple tests within our analysis. This is in agreement with a most recent linkage disequilibrium analysis showing in the haplotype, six SNPs (rs17772159, rs823074, rs803275, rs9438393, rs823090 and rs17772143) but not rs960603 were tightly linked to the lead QTL SNP rs708727 [26]. Our study thus confirms that PM20D1 is an $\mathrm{mQTL}$ mediated primarily by the AD-risk associated SNP (rs708727) as measured in peripheral blood. Furthermore, we exploited the longitudinal data and demonstrated that hypomethylation actually occurs before the symptomatic onset of the disease, conceivably to facilitate increasing expression of the gene to activate its protective function. As disease progresses, methylation level is gradually elevated in most of the CpG sites in the AD patients, which ultimately leads to depletion of the gene transcription and expression. This phenomenon is not observed in the control or MCI groups as their methylation levels are almost constant (Fig. 4). This explains the previously observed hypermethylation in late stage AD patients (Braak staging $\geq 5$ ) in comparison with control. Our models provide a comprehensive picture of the dynamic change of the methylation profile at PM20D1 promoter region, thus complementing the previous work [13]. Of note, the initial methylation decline for these probes actually prevails in $\mathrm{AD}$ vs control for all the stratified genotypes of rs708727 (Additional file 8: Table S8), indicating constant hypomethylation of the promoter region at the early stages of the disease regardless of the patient's disease risk, although it seems that for the AD patients carrying the high-risk SNP, the methylation elevation is faster compared with those with lower risk (Additional file 9: Fig. S3). In our LME model, random slopes of age are not tested due to limited data points per individual which would result in singular fit, or failed converge of the random slope model. By excluding the random slope for the priming manipulation, we assume that the priming effect of age (disease progression) is invariant across subjects in the population. This might be oversimplified and there is possibility that different risk groups possess different rates of increasing methylation level across the PM20D1 promoter region. This hypothesis cannot be directly tested in the current dataset due to the small sample size of AD patients carrying the highrisk SNP $(n=5)$, but it warrants further exploration with larger datasets in the future.

From the U-shape test for the subjects with converted diagnosis, it is identified that 78-79 years old would be the turning point for the methylation level of the probes. This matches the average initial diagnosis age for LOAD at $\sim 78$ years old in the general population [27]. Whether the turning point is triggered by some other factors or solely determined by age is something that requires further investigation. Furthermore, we found a sex-dependent effect on the methylation elevation for most of the probes, indicating that female is at higher risk for hypermethylation of PM20D1 promoter. This reveals yet another possible contributing factor to the females' higher odds for $\operatorname{AD}[28,29]$.

Despite this, hypomethylation does not necessarily translate into higher gene expression. The correlation between methylation profile and gene expression of PM20D1 from cross-sectional data clearly demonstrated that only within the lower-risk genotype carriers, there is an inverse linear relationship. Higher risk genotype carriers' expression profiles are not correlated with methylation, which can be attributed to the inaccessibility of the enhancer to the gene promoter, as suggested by the mechanism model for PM20D1 function in $\mathrm{AD}$. These findings indicate that methylation signatures at the PM20D1 locus are more robustly associated with conversion to AD, than PM20D1 expression. 
The methylation elevation trend after the onset of $\mathrm{AD}$ has been once more observed in brain tissues in the ROSMAP cohort, by using Braak stage as a proxy of the disease's progression. The same correlation pattern between methylation and gene expression for PM20D1 in the brain tissues, as well as the remarkable direct correlation between blood and brain tissue's methylation levels in the third independent cohort at the CpG sites provide strong evidence of a link between peripheral blood methylation profiles and AD-associated methylation differences in the brain tissues at PM20D1 region. Although sex's effect has not been repeated in the brain tissues probably due to the fact the ROSMAP data has been adjusted for age and sex [30], our work illustrates that blood methylation at the PM20D1 promoter region could potentially serve as a surrogate for brain methylation for the study of epigenetics of this gene for AD pathology, along the course of disease evolution.

Of special note, we have also attempted the correlation between PM20D1 methylation level and a few pathological biomarkers, in order to better understand the significance of PMD20D1 methylation in the evolution of AD pathology. For the ROSMAP cohort, methylation level of PM20D1 might be positively associated with amyloid in $\mathrm{AD}$ patients $(p<0.05$ for 3 out of 8 probes in total), but not in MCI (1 probe), nor control (0 probe) or the overall group (1 probe) (Additional file 5: Table S5). For tangles, there is no association of PM20D1 methylation in any of the three diagnostic subgroups, but a weak association in the overall group ( $p<0.05$ for 3 out of 8 probes), probably due to larger sample size and wider range of tangle scores. In the ADNI cohort, there is no correlation with any of the biomarkers (A $31-42$, t-tau and $p$-tau181) from cerebral spinal fluid (CSF) in any of the three diagnostic subgroups, or the overall group (results not shown, biomarker data from [31]), but some positive correlations were found for $\mathrm{p}$-tau181 in plasma, for AD patients only $(p<0.05$ at 3 probes, Additional file 3: Table S3). This is in line with the report that plasma $\mathrm{p}$-tau181 has greater variability than CSF p-tau181 and may be differently regulated depending on $A \beta$ status [32]. Recently, it has been demonstrated that plasma ptau-181 is an indicator of very early brain amyloidosis [33], so the findings imply that PM20D1 could play a role in the metabolism of amyloid proteins, showing correlation with $\mathrm{A} \beta$ biomarkers, but not quantitative tangle scores. Another possible scenario is that soluble amyloid peptide, or other secondary factors associated with very early amyloidosis in the brain, actually triggers hypomethylation of PM20D1 in AD patients, and insoluble plagues in AD stages further affects its methylation level, so the correlation is observed with $A \beta$ deposition.
As a circulating enzyme, PM20D1 regulates a class of $\mathrm{N}$-lipidated amino acids in vivo, and these metabolites function as endogenous uncouplers of mitochondrial respiration [34]. It has been implicated in obesity, type 2 diabetes [34], pain [35] and more recently, Alzheimer's disease [13]. In mouse models, PM20D1 activity was dramatically increased in lipoprotein fractions from APOE-knockout (KO) mice versus wild-type mice. The activation of the PM20D1/N-acyl amino acid pathway is suggested as a contributor to the protection from metabolic and neurological diseases observed in APOE-KO mice [36]. The recent study focusing on PM20D1's role in AD demonstrated that PM20D1 expression is increased both in vitro and in vivo following neurotoxic insults, probably by activating the hypomethylation machinery as illustrated in our work. Forced overexpression of PM20D1 in the hippocampus results in improved learning performance in the mouse model of $\mathrm{AD}$, whereas PM20D1 knockdown increases amyloid plaque load, so it has been suggested to have a protective role against AD. Given that LOAD has also been suggested as a metabolic disorder [37-39], the interplay of PM20D1, more specifically how it operates a protective function against $\mathrm{AD}$, together with other genes implicated in the metabolism for LOAD could help advance our understanding of the disease, and subsequently more efficient hunting for therapeutics.

Accurate, minimally invasive and timely diagnosis of probable AD in living individuals has always been challenging, although most recently great breakthroughs are being made in blood-based biomarkers, such as p-tau181 [32] and p-tau217 [40, 41]. Multiple factors contribute to it, such as heterogenicity of the disease, inaccessibility of the pathological tissues, and lack of robust and readily measurable biomarkers [6]. The dynamic methylation alteration for PM20D1, depicted by the longitudinal data with individuals both before disease onset and following clinical diagnosis opens a probable channel to monitor the disease, and the possibility of a diagnostic tool. Notably, the initial methylation decrease is universal to all the risk-associated SNP genotypes (Additional file 8: Table S8), highlighting its application potential regardless of the genotypes of the SNP locus, thus associated disease risk. The random effect from LME modeling shows that intra-individual methylomic variation at PM20D1 (SD>0.1 for RID) is still non-negligible after controlling fixed effects from age, sex, allelic dosage of rs708727 and rs960603. Notably, APOE status has been initially considered as a covariant in all of our studies, but the effect is not significant $(p>0.1)$, thus not included in the final analysis. While there are presumptively still other factors underlining the baseline methylomic $\beta$ values for PM20D1 promoter, the overall trend of individual 
methylation change, instead of absolute methylation level (which is primarily determined by the SNP allelic dose), is worthy of further evaluation. The current study is the first of its kind applying large-scale longitudinal data to obtain novel insights into the epigenetic signature of PM20D1 intervening with LOAD. Further in-depth study, including those in animal models and patients, as well as its precise relationship with $A \beta$ proteins and $p$-tau levels in blood could help define its sensitivity and specificity to differentiate AD from non-AD and its broader utility down the road.

The preliminary result we observed in the longitudinal data from ADNI cohort is just one piece of evidence that there is correlation for specific pathological changes in AD between different tissues from different cohorts, as manifested at PM20D1 locus. An expansive analysis that addresses DMR and longitudinal data in an integrated model would reveal even more exciting findings; nevertheless, it requires tremendous computational resources and innovative methodologies and tools, which is worth active pursuing. The current study exemplified utilization of the data to distill useful information in the epigenetic landscape of AD. Further exploitation is thus warranted and would be beneficial to the early diagnosis and effective treatment of the formidable disease.

\section{Conclusions}

The longitudinal data from ADNI methylation profiling present compelling evidences demonstrating that changes to the methylome in LOAD detected in blood could reflect pathologic processes implicated in ongoing neurodegeneration in the brains. Specifically to PM20D1 gene locus, it clearly confirms PM20D1 is a methylation QTL essentially coupled to an AD-risk associated SNP rs708727, and illustrates its dynamic role of being hypomethylated in the conversion phase and gradually turning into hypermethylation after onset and during progression of the disease. Our results call for further study, both for PM20D1's role in AD pathology and its potential as a blood-based biomarker, to address the urgent need of early detection and treatment of AD.

\section{Methods}

\section{Study cohort and data source}

ADNI data were all downloaded from the ADNI database (http://adni.loni.usc.edu/). The ADNI was launched in 2003 as a public-private partnership, led by Principal Investigator Michael W. Weiner, MD. The primary goal of ADNI has been to test whether serial magnetic resonance imaging (MRI), positron emission tomography (PET), other biological markers, and clinical and neuropsychological assessment can be combined to measure the progression of mild cognitive impairment $(\mathrm{MCI})$ and early Alzheimer's disease (AD). For up-to-date information, see www.adni-info.org. For details of the methylation profiling procedure, refer to the documentation on the genetic data download page. Briefly, DNA methylation profiling was performed at AbbVie from a total of 1,920 blood samples, including 1719 unique samples and 201 technical replicates (653 unique individuals). Longitudinal DNA samples at baseline and follow-ups were obtained from the cohort. The Illumina Infinium HumanMethylationEPIC BeadChip Array (www.illumina.com), which covers $\sim 866,000 \mathrm{CpGs}$, was used for methylation profiling. After multiple steps of quality check (QC), 1905 samples from 649 subjects were retained and raw methylation data (idat files) were released and analyzed in our study.

Genotype data were obtained from ADNI wholegenome sequencing (WGS) panel, where genotypes were called by the ADNI genetics core using the pipeline following Broad best practices (BWA [42] and GATK HaplotypeCaller [43, 44]). Gene expression data were obtained from blood samples from the 811 participants in the ADNI WGS cohort. The Affymetrix Human Genome U219 Array (Affymetrix (www.affymetrix.com), Santa Clara, CA) was used for expression profiling. The processed gene expression profile was downloaded and used for analysis. Diagnosis data and other demographics (sex, date of birth) were also obtained from ADNI database. Subject selection for the different study sections and the workflow of the ADNI cohort are outlined in Fig. 7a).

All the data from ROSMAP cohort $[45,46]$ were downloaded from Accelerating Medicines Partnership-Alzheimer's Disease (AMP-AD) Knowledge Portal following required guidelines. Methylation $\beta$ values at the interesting CpG probes were obtained from the methylation profile generated on prefrontal cortex samples collected from 740 individuals using the Illumina HumanMethylation450 BeadChip [7]. Genotypes at the interesting loci were extracted from the published WGS data from 1196 subjects [30]. Gene expression Fragments Per Kilobase of transcript per Million mapped reads (FPKM) values were obtained from bulk RNAseq data, where samples were taken from the gray matter of the dorsolateral prefrontal cortex of 724 subjects [30] and the data for 640 subjects were available for download. Clinical and neuropathology data including final diagnosis and Braak stages were matched for each individual and used in the analysis. Subject selection for the different study sections and the workflow of the ROSMAP cohort are outlined in Fig. 7b).

ADNI methylation data quality control and normalization Raw DNA methylation data (idat files) were imported into R, and the Bioconductor package 'bigmelon' [47] was 
used for initial data processing. Detection $\mathrm{p}$ values $(\operatorname{det} \mathrm{P})$, methylation $(\mathrm{M})$ and unmethylation $(\mathrm{uM})$ intensities and $\beta$ values defined as the ratio between the methylated and total signals $(\mathrm{M}+\mathrm{uM})$ were calculated and exported. The ChAMP pipeline [48] for EPIC array was then used for all the downstream analysis following the default workflow. Multiple filtering steps were first applied, by excluding probes with detection $p$ value (default $>0.01$ ), probes with $<3$ beads in at least $5 \%$ of samples per probe, all non-CpG probes contained in the dataset [49], all SNPrelated probes, all multi-hit probes and all probes located in sex chromosomes [50]. Data were then normalized with BMIQ method [51], and batch effects from chip slide were corrected by ComBat [52]. Cell proportion was calculated based on a reference DNA methylation profile, and cell type influence on the whole blood data was removed by RefbaseEWAS [53]. The 'estimateCellCounts' function in minfi [54] was used to estimate the proportional abundance of blood cell types in the each sample based on the intensity of specific for inter-group comparisons as probes present in the EPIC array.

\section{Differential methylated region (DMR) detection}

Since the longitudinal data consist of 649 subjects sampled at dates spanning up to 5 years with 390 subjects having at least 3 visits' data, many of the subjects have changed their diagnostic status over the time course. To focus on the study of LOAD, 70 subjects with sampling age $<65$ at any time point were removed. The diagnosis dates were compared against the blood sample collecting dates for each of the remaining 579 subjects, and the closest diagnosis in time $(<0.5$ years $)$ was chosen as the diagnosis for each individual sample. For clear DMR detection, only those samples keeping a stable diagnosis (i.e., control, $\mathrm{MCI}$, or $\mathrm{AD}$ across all the time points for each subject, respectively) were retained for analysis. This left 424 total subjects for the DMR detection. Among them, 162 were control, 175 were MCI, and the remaining 87 were $\mathrm{AD}$ patients. DMRs were detected using the baseline $\beta$ values by the BumperHunter method implemented in ChAMP [55]. When technical replicates exist for the same sample, one data point was randomly picked. Comparisons were made for AD vs control, AD vs $\mathrm{MCI}$ and $\mathrm{MCI}$ vs control, respectively.

\section{Allelic dosage effect in the DMR region at baseline}

Out of the 424 subjects with stable diagnosis, 423 were genotyped by WGS. Their baseline measurements used for DMR detection were also used for modeling the dosage effect of the two reported associated SNP rs708727 $(\mathrm{GG}=0, \mathrm{GA}=1$, and $\mathrm{AA}=2)$ and $\operatorname{rs} 960603(\mathrm{CC}=0$, $\mathrm{CT}=1$ and $\mathrm{TT}=2$ ). Their effects to the $\beta$ values of the probes at the promoter regions of the PM20D1 gene locus were modeled by the $\mathrm{lm}$ function in $\mathrm{R}$, using age, sex as covariates, stratified by the diagnosis group. The formula, in R syntax, for the model is:

$$
\beta \sim \text { rs708727 + rs960603 + age }+ \text { sex }
$$

\section{Longitudinal data modeling}

Longitudinal analysis was performed on the whole longitudinal data for 540 total subjects with genotypes available from the WGS panel (423 stable diagnosis, 117 converted cases). Their demographics profile is reported in Table 6. For the subjects with a stable diagnosis, linear mixed-effects (LME) models were fit for the $\beta$ values for the probes at the promoter regions of the PM20D1 gene locus, for the 162 controls, $174 \mathrm{MCI}$ patients and $87 \mathrm{AD}$ patients, respectively, by the lmer function in the R package 'ImerTest' [56]. The formula for the linear mixedeffects model is:

$$
\begin{aligned}
\beta & \sim \text { age }+ \text { sex }+ \text { rs } 708727+r s 960603+(1 \mid \text { RID }) \\
& +(1 \mid \text { Slide })+(1 \mid \text { Array })
\end{aligned}
$$

where age, sex and the allelic dosages for the two known SNP rs708727 and rs960603 were modeled as fixed effects, and subject ID (RID), array and slide were modeled as random effects.

The exam dates from plasma p-tau181 data (obtained from recent ADNI release http://adni.loni.usc.edu/ new-longitudinal-plasma-p-tau181-results-available/) were matched against the dates for sample collection of the methylation array, and data from 412 subjects (159 controls, $174 \mathrm{MCI}$ and $79 \mathrm{AD}$ patients) were fit by $\mathrm{LME}$ model using the formula:

$$
\begin{aligned}
\beta & \sim \text { ptau }+ \text { sex }+ \text { rs } 708727+\text { rs } 960603+(1 \mid \text { RID }) \\
& +(1 \mid \text { Slide })+(1 \mid \text { Array })
\end{aligned}
$$

where ptau is log transformed and modeled as fixed effect.

Table 6 Demographic profile for the subjects in the longitudinal data analysis by different diagnosis groups

\begin{tabular}{lllll}
\hline Demographics & \multicolumn{2}{l}{ Stable diagnosis } & Converters \\
\cline { 2 - 4 } & Control & MCI & AD & \\
\hline Total subjects & 162 & 174 & 87 & 117 \\
Male/female & $79 / 83$ & $104 / 70$ & $57 / 30$ & $68 / 49$ \\
\# of visits & $2.66 \pm 0.69$ & $2.63 \pm 0.68$ & $2.10 \pm 0.99$ & $3.10 \pm 0.88$ \\
Age at visits & $77.34 \pm 6.19$ & $75.78 \pm 6.20$ & $79.01 \pm 6.62$ & $78.18 \pm 6.33$ \\
$\begin{array}{l}\text { Duration of } \\
\text { follow-ups }\end{array}$ & $1.71 \pm 0.71$ & $1.73 \pm 0.67$ & $1.12 \pm 1.00$ & $2.07 \pm 0.95$ \\
\hline
\end{tabular}



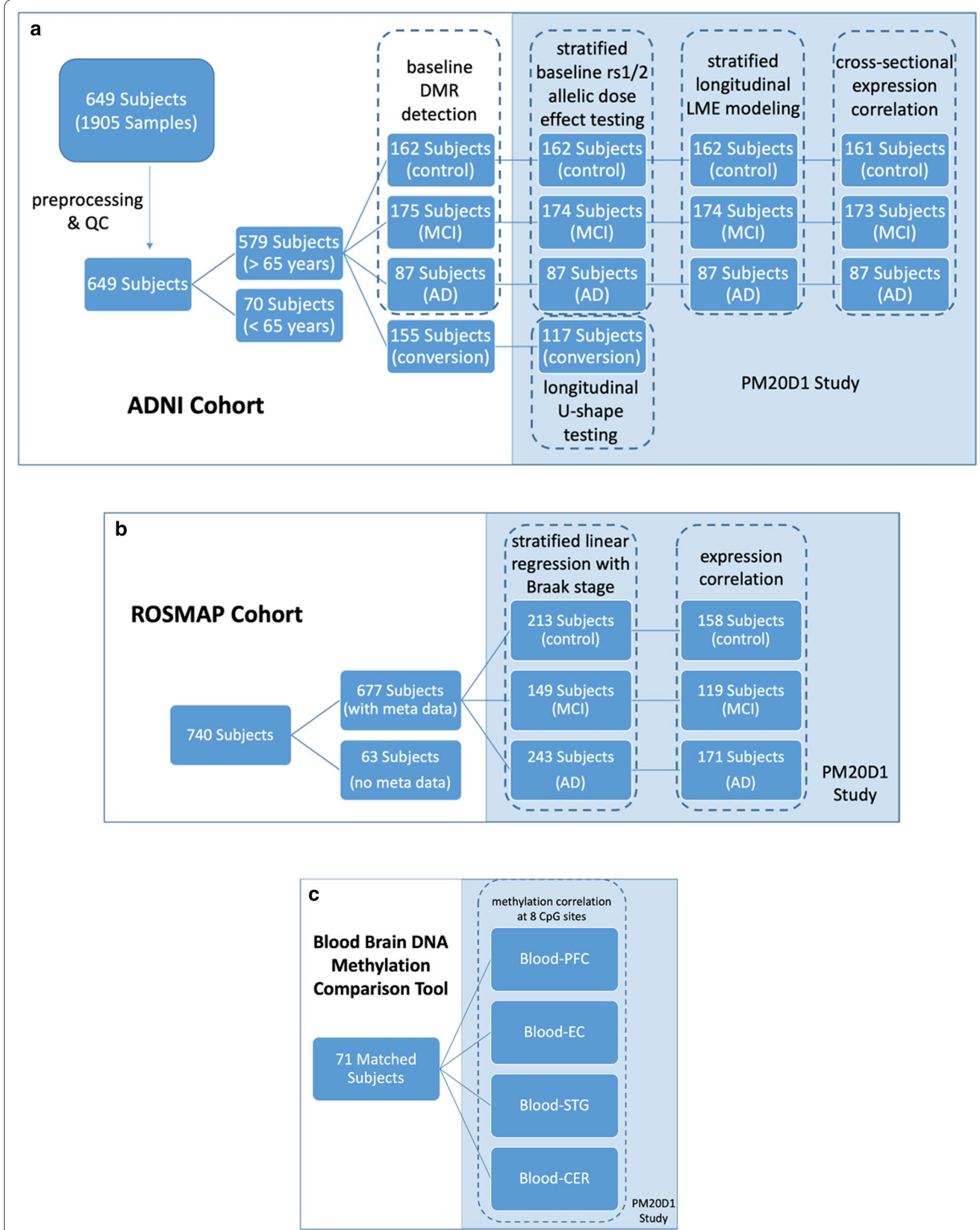

Fig. 7 Workflow and subject selection for the study outlined in this work 
For the group of 117 subjects with changed diagnosis, a U-shape test was carried out by the two-line method introduced by Simonsohn [57] and using the code (http://webstimate.org/twolines/twolines.R), testing if age has a U-shaped effect on $\beta$ values, controlling for sex, allelic dosages of rs708727 and rs960603. The breakpoint is set using the "Robin Hood" algorithm, seeking to obtain higher power to detect a U-shape if it is present [57].

\section{Methylation correlation with gene expression}

For the 423 subjects with stable diagnosis (as well as genotype data), gene expression data were also available from ADNI microarray profiling. All the gene expression data were cross-sectional, and only information on sample collection years is available. The sample collection years were matched against the methylation profiling sample collection years, and those samples collected in the same year were deemed as a matching expression-methylation data pair for each subject. The collection years were more than one year apart between methylation and expression samples for two subjects; thus, they were excluded from this analysis. The relationship between gene expression of PM20D1 and the $\beta$ value for each probe was modeled for the 421 data pairs by the $1 m$ function in R, using age, sex, diagnosis and allelic dosages of rs708727 and rs960603 as covariates, following the equation:

$$
e \sim \beta+a g e+s e x+d x+r s 708727+r s 960603
$$

\section{Methylation data analysis for ROSMAP cohort}

Subjects' clinical diagnosis, Braak stages for brain tissues and demographic information including sex and age at death were obtained from AMP-AD portal. A total of 677 subjects were found to have genotypes, Braak stages and methylation profiles available. Only those subjects with diagnosis as no cognitive impairment (control) or MCI/ AD without any other cause of cognitive impairment $(\operatorname{cogd} x=1,2$ or 4$)$, totaling 605 subjects were included in the final analysis. They were stratified by the diagnosis, and linear regression models in respect to Braak stage, (log transformed) amyloid and (log transformed) tangles were built for the $\beta$ values of the $8 \mathrm{CpG}$ probes at the promoter regions for PM20D1, respectively, controlling for age, sex, allelic dosages of rs708727 and rs960603, using the $\mathrm{lm}$ function in $\mathrm{R}$. Gene expression data were also matched with the methylation data, and 448 subjects out of the 605 were found to have gene expression data from RNASeq profiling. Linear regression models were built between gene expression FPKM value of PM20D1 and the $\beta$ value at each probe for the 448 data pairs by the $\mathrm{lm}$ function in R, using age, sex, diagnosis and allelic dosages of rs708727 and rs960603 as covariates.

\section{DNA methylation correlation between brain tissue} and whole blood

We utilized the Blood Brain DNA Methylation Comparison Tool (https://epigenetics.essex.ac.uk/bloodbrain/), which allows systematic investigation of the correlation of DNA methylation in blood with four brain regions (prefrontal cortex, entorhinal cortex, superior temporal gyrus and cerebellum) from 71 to 75 matched samples in the MRC London Neurodegenerative Disease Brain Bank [8] for all probes present on the Illumina $450 \mathrm{~K}$ Beadchip array [17]. The cohort included both neuropathologically unaffected controls and individuals with variable levels of neuropathology. The data are from published results in 4 dissected brain regions (PFC: $n=114$, EC: $n=108$, STG: $n=117$ and CER: $n=112)$ and matched premortem whole blood samples $(n=80)$ from an overlapping set of 122 individuals [17]. All the correlation values were obtained directly from the database.

\section{Supplementary information}

Supplementary information accompanies this paper at https://doi. org/10.1186/s13148-020-00984-5.

Additional file 1. Table S1: The differentially methylated regions (DMRs) as detected by the comparisons among control, $\mathrm{MCl}$ and AD. Each tab shows the significant regions as ranked by the $p$ values for each comparison.

Additional file 2. Table S2: P values and significant slopes for the regression lines modeled between alternate allelic doses of rs708727/rs960603 and $\beta$ values for the $10 \mathrm{CpG}$ probes at PM20D1 promoter region in the different diagnosis groups.

Additional file 3. Table S3: $P$ values and significant slopes for the regression lines modeled by linear mixed effect modeling between age or p-tau181 and $\beta$ values for the 10 CpG probes at PM20D1 promoter region in the different diagnosis groups.

Additional file 4. Table S4: $P$ values for the regression lines between the $\beta$ values of the $10 \mathrm{CpG}$ probes and the gene expression of PM20D1.

Additional file 5. Table S5: $P$ values for the regression lines between Braak score, amyloid or tangles and $\beta$ values for the $8 \mathrm{CpG}$ probes at PM20D1 promoter region in the different diagnosis groups from the ROSMAP cohort brain samples.

Additional file 6. Table S6: $P$ values for the regression lines between the $\beta$ values of the $8 \mathrm{CpG}$ probes and the gene expression of PM20D1 from the ROSMAP cohort brain samples.

Additional file 7. Table S7: P values and regression coefficients for the correlation between DNA methylation in blood with four brain regions (prefrontal cortex, entorhinal cortex, superior temporal gyrus and cerebellum) from 71 to 75 matched samples for all the $8 \mathrm{CpG}$ probes. The data are taken from the Blood Brain DNA Methylation Comparison Tool [17].

Additional file 8. Table S8: Mean $\beta$ values and their standard deviations (SD) at baseline measurements for the sex and age matched samples at the $10 \mathrm{CpG}$ probes stratified by the allelic dose of rs708727. Control and $\mathrm{MCI}$ samples were obtained by matching AD samples' sex and age using 'matchControls' command in the R package 'e1071.'

Additional file 9. Figure S1. Correlation of methylation $\beta$ values at one of the representative CpG probes (cg14149672) with gene expression of PM20D1 (FPKM) for the ROSMAP cohort brain samples. An overall fit line and the fit lines stratified by the allelic doses of rs708727 are shown. Figure S2. Correlation of DNA methylation in blood with four brain 
regions (prefrontal cortex, entorhinal cortex, superior temporal gyrus and cerebellum) from 71-75 matched samples for one of the representative probes (cg1 1965913). The figure is taken from the Blood Brain DNA Methylation Comparison Tool (17) (https://epigenetics.essex.ac.uk/bloodbrain /index.php?probenameg=cg11965913). Figure S3. Methylation change as disease progresses in the AD patients, modeled by $\beta$ values regressed with age at one of the representative $\mathrm{CpG}$ probes (cg14893161). Scatter plot is colored by the allelic doses of rs 708727 where red $=0$, green $=1$, and blue $=2$. An overall linear fit line, as well as the fit lines for each allelic dose group are also shown.

\section{Abbreviations}

LOAD: Late-onset Alzheimer's disease; QTL: Quantitative trait locus; MCl: Mild cognitive impairment; ADNI: Alzheimer's Disease Neuroimaging Initiative; DMR: Differentially methylated region; LME: Linear mixed effects; ROSMAP: Religious Orders Study and Memory and Aging Project; WGS: Whole-genome sequencing; AMP-AD: Accelerating medicines partnership-Alzheimer's disease: FPKM: Per kilobase of transcript per million mapped reads; SNP: Single-nucleotide polymorphism; CSF: Cerebral spinal fluid; KO: Knock out.

\section{Acknowledgements}

Data collection and sharing for this project was funded by the Alzheimer's Disease Neuroimaging Initiative (ADNI) (National Institutes of Health Grant U01 AG024904) and DOD ADNI (Department of Defense Award Number W81XWH-12-2-0012). ADNI is funded by the National Institute on Aging, the National Institute of Biomedical Imaging and Bioengineering, and through generous contributions from the following: AbbVie, Alzheimer's Association; Alzheimer's Drug Discovery Foundation; Araclon Biotech; BioClinica, Inc.; Biogen; Bristol-Myers Squibb Company; CereSpir, Inc;; Cogstate; Eisai Inc:; Elan Pharmaceuticals, Inc.; Eli Lilly and Company; Eurolmmun; F. Hoffmann-La Roche Ltd and its affiliated company Genentech, Inc;; Fujirebio; GE Healthcare; IXICO Ltd.; Janssen Alzheimer Immunotherapy Research \& Development, LLC.; Johnson \& Johnson Pharmaceutical Research \& Development LLC.; Lumosity; Lundbeck; Merck \& Co., Inc.; Meso Scale Diagnostics, LLC.; NeuroRx Research; Neurotrack Technologies; Novartis Pharmaceuticals Corporation; Pfizer Inc:i Piramal Imaging; Servier; Takeda Pharmaceutical Company; and Transition Therapeutics. The Canadian Institutes of Health Research is providing funds to support ADNI clinical sites in Canada. Private sector contributions are facilitated by the Foundation for the National Institutes of Health (www.fnih. org). The grantee organization is the Northern California Institute for Research and Education, and the study is coordinated by the Alzheimer's Therapeutic Research Institute at the University of Southern California. ADNI data are disseminated by the Laboratory for Neuro Imaging at the University of Southern California.

\section{Authors' contributions}

QW developed the research concept, designed the experiments, analyzed and interpreted the data, and wrote the manuscript. YC, KC and YS contributed to the statistical analysis. BR contributed to the experiment design and scientific interpretation. ER and JD jointly supervised the project. All authors read and approved the final manuscript.

\section{Funding}

QW is supported in part by PG08973 from Arizona State University. YC, YS, KC, and EMR are supported in part by R01AG031581, P30AG019610, DHS and the State of Arizona. Arizona Department of Health Services (ADHS), United States Grant No. CTR040636 (previously ADHS Grant No. ADHS14-052688). The funding sources did not play a role in study design, the collection, analysis and interpretation of data, writing of the report; or in the decision to submit the article for publication.

\section{Availability of data and materials}

The datasets supporting the conclusions of this article are available in the following repositories: ANDI: http://adni.loni.usc.edu/data-samples/access-data/. ROSMAP: https://www.synapse.org/\#!Synapse:syn3219045 All the analysis results and codes are available from the authors upon request.

\section{Ethics approval and consent to participate} Not applicable.

\section{Consent for publication}

Not applicable.

\section{Competing interests}

The authors declare that they have no competing interests.

\section{Author details}

${ }^{1}$ ASU-Banner Neurodegenerative Disease Research Center, Arizona State University, Tempe, AZ, USA. ${ }^{2}$ Banner Alzheimer's Institute, Phoenix, AZ, USA.

${ }^{3}$ Icahn School of Medicine at Mount Sinai, New York, NY, USA.

Received: 25 June 2020 Accepted: 18 November 2020

Published online: 09 December 2020

\section{References}

1. Kunkle BW, Grenier-Boley B, Sims R, Bis JC, Damotte V, Naj AC, et al. Genetic meta-analysis of diagnosed Alzheimer's disease identifies new risk loci and implicates Abeta, tau, immunity and lipid processing. Nat Genet. 2019;51(3):414-30.

2. Sanchez-Mut JV, Graff J. Epigenetic alterations in Alzheimer's disease. Front Behav Neurosci. 2015;9:347.

3. Liu X, Jiao B, Shen L. The epigenetics of Alzheimer's disease: factors and therapeutic implications. Front Genet. 2018;9:579.

4. Esposito M, Sherr GL. Epigenetic modifications in alzheimer's neuropathology and therapeutics. Front Neurosci. 2019;13:476.

5. Levenson W. DNA methylation as a universal biomarker. Expert Rev Mol Diagn. 2010;10(4):481-8.

6. Fransquet PD, Lacaze P, Saffery R, McNeil J, Woods R, Ryan J. Blood DNA methylation as a potential biomarker of dementia: a systematic review. Alzheimers Dement. 2018;14(1):81-103.

7. De Jager PL, Srivastava G, Lunnon K, Burgess J, Schalkwyk LC, Yu L, et al. Alzheimer's disease: early alterations in brain DNA methylation at ANK1, BIN1, RHBDF2 and other loci. Nat Neurosci. 2014;17(9):1156-63.

8. Lunnon K, Smith R, Hannon E, De Jager PL, Srivastava G, Volta M, et al. Methylomic profiling implicates cortical deregulation of ANK1 in Alzheimer's disease. Nat Neurosci. 2014;17(9):1164-70.

9. Graff J, Rei D, Guan JS, Wang WY, Seo J, Hennig KM, et al. An epigenetic blockade of cognitive functions in the neurodegenerating brain. Nature. 2012;483(7388):222-6.

10. Gasparoni G, Bultmann S, Lutsik P, Kraus TFJ, Sordon S, Vlcek J, et al. DNA methylation analysis on purified neurons and glia dissects age and Alzheimer's disease-specific changes in the human cortex. Epigenetics Chromatin. 2018;11(1):41.

11. Smith RG, Hannon E, De Jager PL, Chibnik L, Lott SJ, Condliffe D, et al. Elevated DNA methylation across a 48-kb region spanning the HOXA gene cluster is associated with Alzheimer's disease neuropathology. Alzheimers Dement. 2018;14(12):1580-8.

12. Altuna M, Urdanoz-Casado A, Sanchez-Ruiz de Gordoa J, Zelaya MV, Labarga A, Lepesant JMJ, et al. DNA methylation signature of human hippocampus in Alzheimer's disease is linked to neurogenesis. Clin Epigenetics. 2019;11(1):91.

13. Sanchez-Mut JV, Heyn H, Silva BA, Dixsaut L, Garcia-Esparcia P, Vidal E, et al. PM20D1 is a quantitative trait locus associated with Alzheimer's disease. Nat Med. 2018;24(5):598-603.

14. Kaut O, Ramirez A, Pieper H, Schmitt I, Jessen F, Wullner U. DNA methylation of the TNF-alpha promoter region in peripheral blood monocytes and the cortex of human Alzheimer's disease patients. Dement Geriatr Cogn Disord. 2014;38(1-2):10-5.

15. Yu L, Chibnik LB, Yang J, McCabe C, Xu J, Schneider JA, et al. Methylation profiles in peripheral blood CD4+ lymphocytes versus brain: the relation to Alzheimer's disease pathology. Alzheimers Dement. 2016;12(9):942-51.

16. Smith AK, Kilaru V, Kocak M, Almli LM, Mercer KB, Ressler KJ, et al. Methylation quantitative trait loci (meQTLs) are consistently detected across ancestry, developmental stage, and tissue type. BMC Genomics. 2014;15:145.

17. Hannon E, Lunnon K, Schalkwyk L, Mill J. Interindividual methylomic variation across blood, cortex, and cerebellum: implications for epigenetic 
studies of neurological and neuropsychiatric phenotypes. Epigenetics. 2015;10(11):1024-32.

18. Teschendorff AE, Menon U, Gentry-Maharaj A, Ramus SJ, Gayther SA, Apostolidou S, et al. An epigenetic signature in peripheral blood predicts active ovarian cancer. PLoS ONE. 2009;4(12):e8274.

19. Muller-Ehrenberg L, Riphagen JM, Verhey FRJ, Sack AT, Jacobs HIL, Alzheimer's disease neuroimaging I. Alzheimer's disease biomarkers have distinct associations with specific hippocampal subfield volumes. J Alzheimers Dis. 2018:66(2):811-23.

20. Gibbs JR, van der Brug MP, Hernandez DG, Traynor BJ, Nalls MA, Lai SL, et al. Abundant quantitative trait loci exist for DNA methylation and gene expression in human brain. PLoS Genet. 2010;6(5):e1000952.

21. Holtzman DM, Morris JC, Goate AM. Alzheimer's disease: the challenge of the second century. Sci Transl Med. 2011;3(77):77sr1.

22. Sanchez-Mut JV, Glauser L, Monk D, Graff J. Comprehensive analysis of PM20D1 QTL in Alzheimer's disease. Clin Epigenetics. 2020;12(1):20.

23. Kim B, Choi Y, Kim HS, Im HI. Methyl-CpG binding protein 2 in Alzheimer dementia. Int Neurourol J. 2019;23(Suppl 2):S72-81.

24. Gunasekara CJ, Scott CA, Laritsky E, Baker MS, MacKay H, Duryea JD, et al. A genomic atlas of systemic interindividual epigenetic variation in humans. Genome Biol. 2019;20(1):105.

25. McRae AF, Marioni RE, Shah S, Yang J, Powell JE, Harris SE, et al. Identification of 55,000 replicated DNA methylation QTL. Sci Rep. 2018;8(1):17605.

26. Benson KK, Hu W, Weller AH, Bennett AH, Chen ER, Khetarpal SA, et al. Natural human genetic variation determines basal and inducible expression of PM20D1, an obesity-associated gene. Proc Natl Acad Sci USA. 2019;1 16(46):23232-42.

27. Yeh TS, Wang JD, Ku LJ. Estimating life expectancy and lifetime healthcare costs for Alzheimer's disease in Taiwan: does the age of disease onset matter? J Alzheimers Dis. 2019.

28. Farrer LA, Cupples LA, Haines JL, Hyman B, Kukull WA, Mayeux R, et al. Effects of age, sex, and ethnicity on the association between apolipoprotein E genotype and Alzheimer disease. A meta-analysis. APOE and Alzheimer Disease Meta Analysis Consortium. JAMA. 1997;278(16):1349-56

29. Ferretti MT, Iulita MF, Cavedo E, Chiesa PA, Schumacher Dimech A, Santuccione Chadha A, et al. Sex differences in Alzheimer disease: the gateway to precision medicine. Nat Rev Neurol. 2018;14(8):457-69.

30. De Jager PL, Ma Y, McCabe C, Xu J, Vardarajan BN, Felsky D, et al. A multiomic atlas of the human frontal cortex for aging and Alzheimer's disease research. Sci Data. 2018:5:180142.

31. Toledo JB, Xie SX, Trojanowski JQ, Shaw LM. Longitudinal change in CSF Tau and Abeta biomarkers for up to 48 months in ADNI. Acta Neuropathol. 2013;126(5):659-70.

32. Janelidze $S$, Mattsson N, Palmqvist S, Smith R, Beach TG, Serrano GE, et al. Plasma P-tau181 in Alzheimer's disease: relationship to other biomarkers, differential diagnosis, neuropathology and longitudinal progression to Alzheimer's dementia. Nat Med. 2020;26(3):379-86.

33. Karikari TK, Pascoal TA, Ashton NJ, Janelidze S, Benedet AL, Rodriguez $J$, et al. Blood phosphorylated tau 181 as a biomarker for Alzheimer's disease: a diagnostic performance and prediction modelling study using data from four prospective cohorts. Lancet Neurol. 2020;19(5):422-33.

34. Long JZ, Svensson KJ, Bateman LA, Lin H, Kamenecka T, Lokurkar IA, et al. The secreted enzyme PM20D1 regulates lipidated amino acid uncouplers of mitochondria. Cell. 2016;166(2):424-35.

35. Long JZ, Roche AM, Berdan CA, Louie SM, Roberts AJ, Svensson KJ, et al. Ablation of PM20D1 reveals $\mathrm{N}$-acyl amino acid control of metabolism and nociception. Proc Natl Acad Sci USA. 2018;115(29):E6937-45.

36. Kim JT, Jedrychowski MP, Wei W, Fernandez D, Fischer CR, Banik SM, et al. A plasma protein network regulates PM20D1 and N-acyl amino acid bioactivity. Cell Chem Biol. 2020.

37. Merlo S, Spampinato S, Canonico PL, Copani A, Sortino MA. Alzheimer's disease: brain expression of a metabolic disorder? Trends Endocrinol Metab. 2010;21(9):537-44.

38. Clarke JR, Ribeiro FC, Frozza RL, De Felice FG, Lourenco MV. Metabolic dysfunction in Alzheimer's disease: from basic neurobiology to clinical approaches. J Alzheimers Dis. 2018:64(s1):S405-26.
39. Butterfield DA, Halliwell B. Oxidative stress, dysfunctional glucose metabolism and Alzheimer disease. Nat Rev Neurosci. 2019;20(3):148-60.

40. Barthelemy NR, Horie K, Sato C, Bateman RJ. Blood plasma phosphorylated-tau isoforms track CNS change in Alzheimer's disease. J Exp Med. 2020;217(11)

41. Palmqvist S, Janelidze S, Quiroz YT, Zetterberg H, Lopera F, Stomrud E, et al. Discriminative accuracy of plasma phospho-tau217 for Alzheimer disease versus other neurodegenerative disorders. JAMA. 2020.

42. Li H, Durbin R. Fast and accurate short read alignment with BurrowsWheeler transform. Bioinformatics. 2009;25(14):1754-60.

43. DePristo MA, Banks E, Poplin R, Garimella KV, Maguire JR, Hartl C, et al. A framework for variation discovery and genotyping using next-generation DNA sequencing data. Nat Genet. 2011;43(5):491-8.

44. Van der Auwera GA, Carneiro MO, Hartl C, Poplin R, Del Angel G, LevyMoonshine A, et al. From FastQ data to high confidence variant calls: the Genome Analysis Toolkit best practices pipeline. Curr Protoc Bioinform. 2013;43:11 0 1-33.

45. Bennett DA, Schneider JA, Arvanitakis Z, Wilson RS. Overview and findings from the religious orders study. Curr Alzheimer Res. 2012;9(6):628-45.

46. Bennett DA, Schneider JA, Buchman AS, Barnes LL, Boyle PA, Wilson RS. Overview and findings from the rush Memory and Aging Project. Curr Alzheimer Res. 2012;9(6):646-63.

47. Gorrie-Stone TJ, Smart MC, Saffari A, Malki K, Hannon E, Burrage J, et al. Bigmelon: tools for analysing large DNA methylation datasets. Bioinformatics. 2019;35(6):981-6.

48. Tian Y, Morris TJ, Webster AP, Yang Z, Beck S, Feber A, et al. ChAMP: updated methylation analysis pipeline for Illumina BeadChips. Bioinformatics. 2017;33(24):3982-4.

49. Chen YA, Lemire M, Choufani S, Butcher DT, Grafodatskaya D, Zanke BW, et al. Discovery of cross-reactive probes and polymorphic CpGs in the Illumina Infinium HumanMethylation450 microarray. Epigenetics. 2013;8(2):203-9.

50. Zhou W, Laird PW, Shen H. Comprehensive characterization, annotation and innovative use of Infinium DNA methylation BeadChip probes. Nucleic Acids Res. 2017:45(4):e22.

51. Teschendorff AE, Marabita F, Lechner M, Bartlett T, Tegner J, GomezCabrero $D$, et al. A beta-mixture quantile normalization method for correcting probe design bias in Illumina Infinium 450 k DNA methylation data. Bioinformatics. 2013;29(2):189-96.

52. Johnson WE, Li C, Rabinovic A. Adjusting batch effects in microarray expression data using empirical Bayes methods. Biostatistics. 2007:8(1):118-27.

53. Houseman EA, Accomando WP, Koestler DC, Christensen BC, Marsit CJ, Nelson $\mathrm{HH}$, et al. DNA methylation arrays as surrogate measures of cell mixture distribution. BMC Bioinform. 2012;13:86.

54. Aryee MJ, Jaffe AE, Corrada-Bravo H, Ladd-Acosta C, Feinberg AP, Hansen $K D$, et al. Minfi: a flexible and comprehensive Bioconductor package for the analysis of Infinium DNA methylation microarrays. Bioinformatics. 2014;30(10):1363-9.

55. Jaffe $A E$, Murakami P, Lee H, Leek JT, Fallin MD, Feinberg AP, et al. Bump hunting to identify differentially methylated regions in epigenetic epidemiology studies. Int J Epidemiol. 2012;41(1):200-9.

56. Kuznetsova A, Brockhoff PB, Christensen RHB. Imertest package: tests in linear mixed effects models. J Stat Softw. 2017;82(13).

57. Simonsohn U. Two lines: a valid alternative to the invalid testing of U-shaped relationships with quadratic regressions. Adv Methods Pract Psychol Sci. 2018;1(4):538-55.

\section{Publisher's Note}

Springer Nature remains neutral with regard to jurisdictional claims in published maps and institutional affiliations. 